\title{
Genes of the most conserved WOX clade in plants affect root and flower development in Arabidopsis
} Yves Deveaux ${ }^{1}$, Claire Toffano-Nioche ${ }^{1}$, Gaelle Claisse ${ }^{1}$, Vincent Thareau ${ }^{1}$, Halima Morin ${ }^{2}$, Patrick Laufs ${ }^{2}$, Hervé Moreau ${ }^{3}$, Martin Kreis*1 and Alain Lecharny ${ }^{1}$

Address: ${ }^{1}$ Université Paris-Sud 11, Institut de Biotechnologie des Plantes, Bâtiment 630, UMR/CNRS 8618, F-91405 Orsay, France, ${ }^{2}$ Laboratoire de Biologie Cellulaire, Institut J. P. Bourgin, INRA, 78026 Versailles Cedex, France and ${ }^{3}$ Observatoire Océanologique, Laboratoire Arago, Unité Mixte de Recherche 7628, CNRS-Université Pierre et Marie Curie, BP44, 66651 Banyuls sur Mer Cedex, France

Email: Yves Deveaux - yves.deveaux@u-psud.fr; Claire Toffano-Nioche - claire.toffano-nioche@u-psud.fr; Gaelle Claisse - gaelle.claisse@upsud.fr; Vincent Thareau - vincent.thareau@u-psud.fr; Halima Morin - Halima.Morin@versailles.inra.fr;

Patrick Laufs - Patrick.Laufs@versailles.inra.fr; Hervé Moreau - herve.moreau@obs-banyuls.fr; Martin Kreis* - martin.kreis@u-psud.fr; Alain Lecharny - alain.lecharny@u-psud.fr

* Corresponding author

Published: 24 October 2008

BMC Evolutionary Biology 2008, 8:29| doi:|0.|I86/I47|-2|48-8-29|
Received: 23 May 2008

Accepted: 24 October 2008

This article is available from: http://www.biomedcentral.com/I47I-2/48/8/29I

(C) 2008 Deveaux et al; licensee BioMed Central Ltd.

This is an Open Access article distributed under the terms of the Creative Commons Attribution License (http://creativecommons.org/licenses/by/2.0), which permits unrestricted use, distribution, and reproduction in any medium, provided the original work is properly cited.

\begin{abstract}
Background: The Wuschel related homeobox (WOX) family proteins are key regulators implicated in the determination of cell fate in plants by preventing cell differentiation. A recent WOX phylogeny, based on WOX homeodomains, showed that all of the Physcomitrella patens and Selaginella moellendorffii WOX proteins clustered into a single orthologous group. We hypothesized that members of this group might preferentially share a significant part of their function in phylogenetically distant organisms. Hence, we first validated the limits of the WOXI3 orthologous group (WOXI3 OG) using the occurrence of other clade specific signatures and conserved intron insertion sites. Secondly, a functional analysis using expression data and mutants was undertaken.

Results: The WOXI3 OG contained the most conserved plant WOX proteins including the only WOX detected in the highly proliferating basal unicellular and photosynthetic organism Ostreococcus tauri. A large expansion of the WOX family was observed after the separation of mosses from other land plants and before monocots and dicots have arisen. In Arabidopsis thaliana, AtWOXI3 was dynamically expressed during primary and lateral root initiation and development, in gynoecium and during embryo development. AtWOX 13 appeared to affect the floral transition. An intriguing clade, represented by the functional AtWOXI 4 gene inside the WOXI3 OG, was only found in the Brassicaceae. Compared to AtWOXI3, the gene expression profile of AtWOXI4 was restricted to the early stages of lateral root formation and specific to developing anthers. A mutational insertion upstream of the AtWOXI 4 homeodomain sequence led to abnormal root development, a delay in the floral transition and premature anther differentiation.

Conclusion: Our data provide evidence in favor of the WOXI3 OG as the clade containing the most conserved WOX genes and established a functional link to organ initiation and development in Arabidopsis, most likely by preventing premature differentiation. The future use of Ostreococcus tauri and Physcomitrella patens as biological models should allow us to obtain a better insight into the functional importance of WOXI3 OG genes.
\end{abstract}




\section{Background}

Homeodomain (HD) containing transcription factors are key regulators implicated in the determination of cell fate and cell differentiation in both plants and animals. In Angiosperms, a gene called WUSCHEL (WUS) has been isolated from many different species. WUS was the first identified member of the Wuschel-related homeobox (WOX) subfamily $[1,2]$ that is only found in plants.

The specific expression of the WOX genes in different plant organs and cell types [2-7] suggested an important role for them during organogenesis. The WUS gene is expressed in a restricted region of the meristem called the organizing centre located below the stem cells of the shoot apical meristem (SAM) and functions non-cell autonomously to control the stem cell fate [8]. Interestingly Gallois et al [3] showed recently that ectopic expression of WUS in the root establishes shoot stem cells and leaf development. Other members of the WOX family might also prevent premature cell differentiation in developing organs or tissues. Recently, WOX5 was shown to be involved in stem cell maintenance signaling in the root [9]. Wu et al [10] have shown that WOX9 (Stimpy/STIP) prevents premature cell differentiation during organ growth, in addition to contributing positively to WUS expression. This work also revealed that stip mutants can be rescued by sucrose, a modulator of cell proliferation trough cyclin D induction [11,12]. Two other WOX genes, named Narrow Sheath 1 and 2, that are maize orthologues of Arabidopsis thaliana AtWOX6 were shown to be involved in the recruitment of the lateral founder cells within the SAM prior to the primordia development [13]. Last, Haecker et al [1] analyzed the expression dynamics of the WOX genes during $A$. thaliana embryo development and showed that members of the WOX family mark cell fate decisions during embryonic patterning. Taken together, these results suggest that not only WUS but several other WOX genes play a role in regulating cell division and preventing cell differentiation.

Characterized genomes of Angiosperms contain more than 10 different WOXs and phylogenetic studies suggest that the WOX family existed in the last common ancestor of monocots and core eudicots $[14,15]$. A recent WOX phylogeny based on WOX HDs from different Angiosperms, the moss Physcomitrella patens and the lycophyte Selaginella moellendorffii showed that all of the $P$. patens and eight of the $S$. moellendorffii WOX genes clustered in the same clade [16]. Indeed, the phylogenetic analyses of a transcription factor family like WOX suffer from some limitations due to the relatively short length of the HD, which is the only conserved sequence between the WOX protein family members, and the relatively few land plant species with a completely characterized proteome. As a consequence, the patterns of phylogenic trees of large families of plant transcription factors are often weakly supported by statistics and may be affected by long branch attraction [17]. Nevertheless, clade specific gene features and protein domains were observed in transcription factors $[18,19]$. Thus, in this study, we used clade specific signatures beyond the HD and clade specific conservation of intron insertion sites, to provide additional data in support of the orthology links predicted by the WOX phylogeny. In order to carry out the latter analyses, complete genome sequences with accurate structural annotations are essential. Our results strongly support the existence of a WOX13 orthologous group (WOX13 OG) that, contrary to the two other WOX OGs, contains genes from many different members of the plant kingdom, including basal species, and a branch apparently specific to Brassicaceae. To obtain information on the function of the genes belonging to the WOX13 clade, the cell specificity of At WOX13 and At WOX14 expression was established experimentally and phenotypes resulting from mutations within these two genes were characterized in the plant model A. thaliana.

\section{Results \\ A WOX distance tree from model genomes}

We carefully screened several completely sequenced eukaryote genomes for WOX genes, i.e., two Angiosperms, A. thaliana and $O$. sativa, three algae, O. tauri, O. lucimarinus and Chlamydomonas reinhardtii and two yeasts, Saccharomyces cerevisiae and Schizosaccharomyces pombe. No WOX genes were identified outside of the plant kingdom and Ostreococcus revealed only one WOX gene per genome. Moreover, using the HMMsearch tool [20], we found only one HD gene in C. reinhardtii, 2 in S. pombe, 4 in O. tauri and 8 in $S$. cerevisiae compared to the 92 HD genes in $A$. thaliana.

Distance and phylogenic trees were computed from the HD sequences of the WOX proteins from the five plant model genomes, $O$. tauri, $P$. patens, $S$. moellendorffii, A. thaliana and $O$. sativa (see method section). The clustering of the WOX proteins in 3 different OGs were consistent using either the Neighbor-Joining (Figure 1) or the Maximum likelihood (see additional file 1: Figure 1) methods and different outgroups. The topology of the trees clearly showed three different OGs namely the WOX1, WOX8 and WOX13 MOGs (for Model plant OG). Each OG contained rice and Arabidopsis proteins, but the single WOX protein of O. tauri, the three P. patens and the three S. Moellendorffii WOX proteins were all found in the same group, the WOX13 MOG. The WOX1 MOG contained AtWOX17 and AtWUS, the WOX8 OG associated AtWOX8, 9, 11 and 12 and the WOX13 OG included AtWOX10, 13 and 14. Thus, the previous observation of the presence of the $P$. patens proteins only in the WOX13 OG group was extended to $O$. tauri and $S$. moellendorffii. However, the 


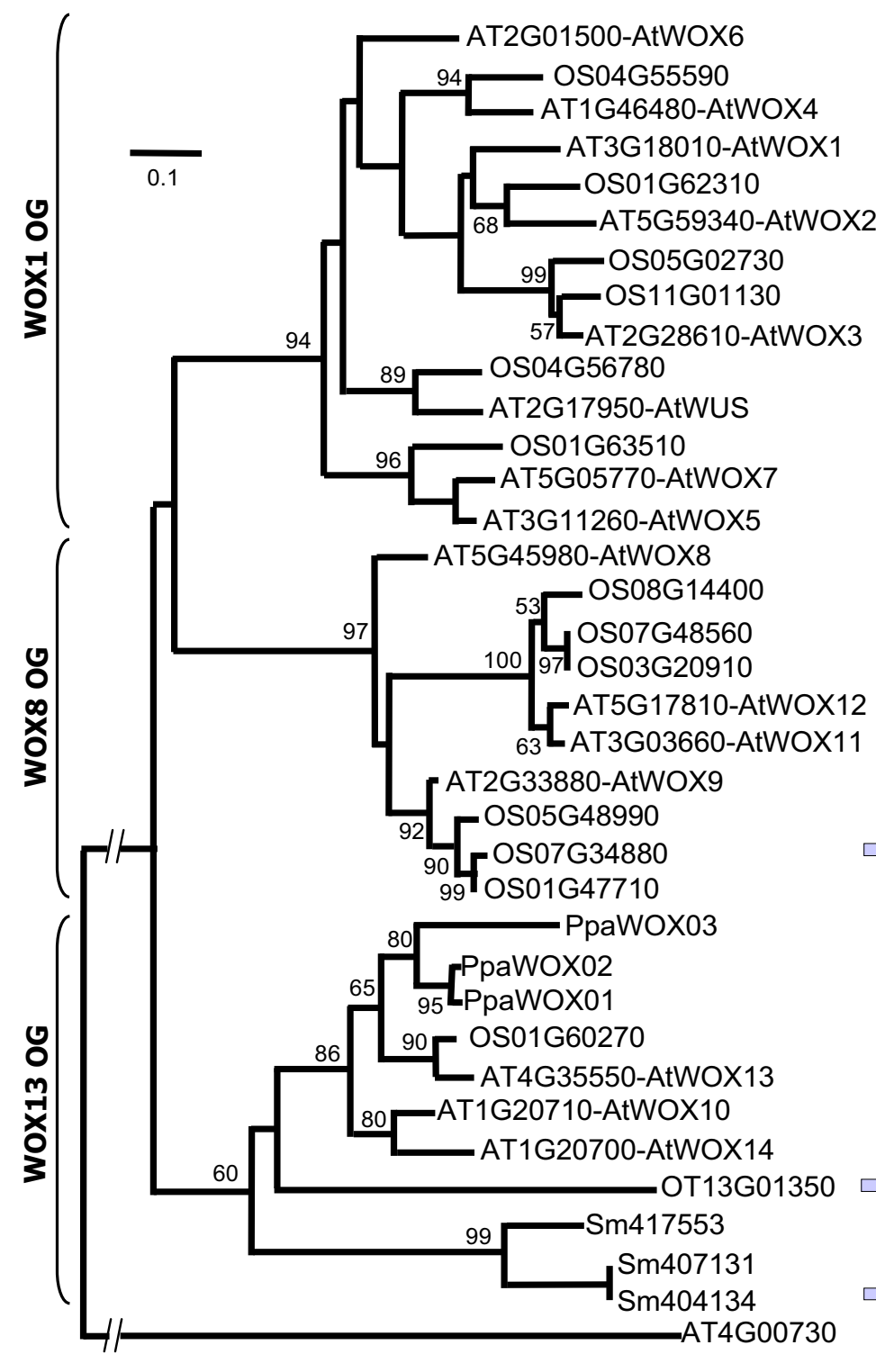

Homeodomain WOX (60 aa)
TLxLFP WOX1 MOG (10aa)

phase 0 intron
WUS motif (8aa)

WOX13 MOG (39 aa)
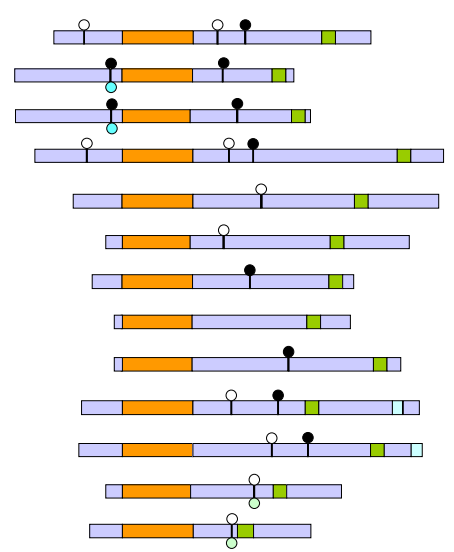

$\square \quad$,
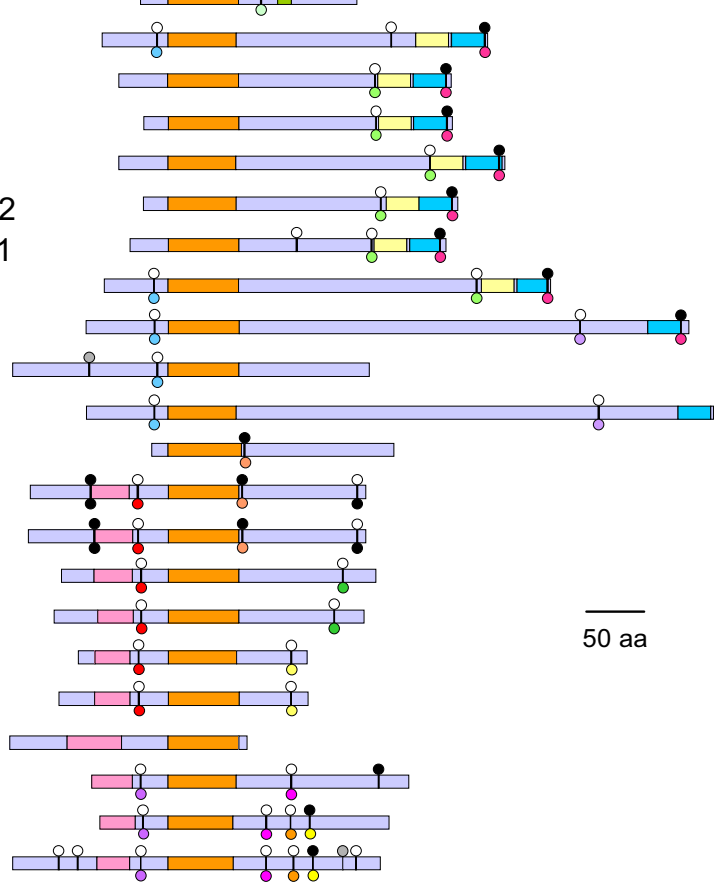

\begin{tabular}{llll}
\hline 11 & 0 & 0 & 0
\end{tabular}

VFIN WOX8 MOG (30 aa)

LQXG WOX8 MOG (30 aa)

Figure I

WOX genes from model plant genomes. The neighbor-joining tree of the WOX proteins from the 5 completely sequenced genomes of the green lineage: Arabidopsis thaliana (AT prefix), Oryza sativa (OS prefix), Physcomitrella patens (Ppa prefix), Ostreococcus tauri (OT prefix) and Selaginella moellendorffii (Sm prefix). The tree topology was inferred from Jones-Taylor-Thornton matrix distances. The significance of each node was tested using 1000 bootstrap replicates. Only bootstrap values above $50 \%$ are shown. The scale, at the top left of the tree, indicates 0.1 substitutions per site. Different structural features are represented by different signs and colors as described at the bottom of the figure. 
introduction of OtWOX and the SmWOXs did not change the main branching of the trees nor significantly modified the bootstrap values at the internal node of the tree. The 14 available WOX sequences from different Gymnosperms were distributed between the three OGs (data not shown). Furthermore, the trees we obtained were not consistent with the phylogenetic tree of plant species as it is now known [21]. Thus the WOX trees we produced suggest probably several rounds of gene deletions in O. tauri and $P$. patens in order to explain the branching of the WOX13 OG. The finding that all the WOX sequences of $O$. tauri and $P$. patens, as well as those of $S$. moellendorffii were clustered in the WOX13 OG suggested to us that AtWOX10, 13, 14 might represent the most interesting genes to study the functional diversification of the WOX genes although they are the WOX genes for which we have the least functional information (reviewed in [22]). This prompted us to look at the specificity of the WOX13 OG with regards to gene structure and conserved amino acid signatures other than the HD. Conservation in gene structure is an indication of a common ancestor while conserved amino acid signatures are linked to functions such as protein-protein interactions or regulatory sites.

\section{WOX gene structures}

The intron-exon organization of WOX genes from the model genomes showed different gene structures (see additional file 2: Figure 1). Alignments supporting the conservation of intron insertion sites are given in additional file 2: Figure 2. The organization of the gene structures along the phylogeny tree did not reveal any contradiction between gene structures and tree pattern. Intron site conservations were exclusively observed within each of the three OGs and never between them. In this context, it was interesting to observe that the insertion site of the first intron in AtWOX13 is conserved in almost all the genes (from moss to Angiosperms) belonging to the WOX13 OG. The only exception was PpaWOX03, a possible pseudogene (see below). This observation cannot be extended to $O$. tauri as its genes do not have introns. $S$. moellendorffii genes have introns and the three full-length SmWOXs have one conserved intron insertion site downstream from the WOX13 motif. The latter intron of SmWOXs is in a similar position (relative to the coding region) to the first highly conserved intron of WOX13 OG genes in moss and Angiosperms. Nevertheless, there is no trace of amino acid sequence conservation in the corresponding protein region between $S$. moellendorffii and the other genes in the WOX13 OG (see additional file 3: Table 3 ). When taken together, our analyses of intron insertion sites support the boundaries of the WOX13 OG as defined by phylogeny. In addition to the highly conserved intron upstream of the HD coding sequence found in the WOX13 OG, AtWOX10 and AtWOX14 share another conserved intron insertion site downstream of the HD while
OS01G60270 and AtWOX13 share a different intron insertion site in the 3 ' region of the gene. The tree pattern implies first a loss of the orthologues of AtWOX10 and 14 in O. sativa and in P. patens and secondly that AtWOX13 and $0 S 01 G 60270$ are orthologous. Interestingly, the two latter genes share a specific intron insertion site.

\section{Specific motifs in the WOX proteins}

A search for specific motifs other than the HD used to build the distance and phylogeny trees led us to identify HMMs that were specific to each clade and located either within the $\mathrm{N}$ or the $\mathrm{C}$ terminal regions of the different proteins. These HMMs are illustrated by sequence logos (see additional file 2: Figure 3 ) and by alignments (see additional file 2: Figure 4). Only proteins of the WOX1 clade have a small motif of 10 amino acids (i.e., the TLxLFP motif) in the C-terminal region, but at relatively variable distances from the HD. TLxLFP has previously been described as a characteristic motif of WUS proteins $[1,23]$, but we show here that it is common to the entire WOX1 clade, i.e., it is observed not only in WUS, but also in the AtWOX1-7 proteins. However, the WUS proteins of $A$. thaliana (AT2G17950-AtWUS) and $O$. sativa (OS04G56780) share specific features not observed in any of the other proteins of the WOX1 MOG. Indeed, they display 1) the LELxL WUS motif [1] known as EAR-like or ERF-like motifs that are located in the outmost C-terminal region, similar to a motif found in potent transcriptional repressors in plants i.e., Superman [24] and Aux/IAA [25]; 2 ) the same intron exon structure in the coding regions of each gene; and 3 ) an additional amino acid at a conserved position in the homeodomain.

Nearly all proteins of the WOX8 MOG contain two contiguous motifs of 30 amino acids situated near to their Cterminus (i.e., VFIN-WOX8 and LQxG-WOX8 MOG motifs). The latter motifs are surrounded by two conserved introns. Remarkably, these introns are also found in OS05G48990 in which the VFIN-WOX8 MOG motif is replaced by a non-conserved sequence of a similar length.

All the proteins of the WOX13 MOG, except for PpaWOX03, share a 39 amino acid motif that is found upstream from the HD and named the WOX13 MOG motif. This observation provides further support for the validity of the WOX13 OG as defined by phylogeny and to the hypothesis of a specific function of the genes clustered within. For all of these reasons, it was decided to investigate further the WOX13 clade.

\section{WOXI3 OG distance tree}

Thirty three full length WOX proteins were used to reconstruct the distance tree of the WOX13 clade (Figure 2), using OtWOX as the outgroup. The neighbor-joining tree showed two main branches. The first branch contained all 
of the Gymnosperm and the moss WOXs. The second branch contained AtWOX13 together with many Angiosperm sequences. This branch was further separated into two branches, one contained AtWOX13 and many proteins from different Angiosperms while the second contained AtWOX10, AtWOX14 and a Brassica rapa sequence.

The preferential presence of amino acid motifs fully supported the separation of the different gene groups within the trees. Obviously, all proteins of the WOX13 clade displayed the WOX HD and the WOX13 motif. Moreover, we also identified specific motifs for the AtWOX10, 14 and for the AtWOX13 branches (see additional file 2: Figure $3)$. All Angiosperm proteins located inside the WOX13 OG shared two motifs, the YxDpl-WOX13 motif located between the WOX13 MOG motif and the HD, and the ESExE-WOX13 motif just downstream from the HD. The monocots of the AtWOX13 branch also share a specific motif called the monocot-WOX13 motif that was detected also in two other partial proteins of two monocotyledon species.

Proteins of the AtWOX10, 14 branch shared two specific motifs, the YFdPM-WOX10 and QAdDaAVTT-WOX10 motifs. They were located to the same region of the protein as the YxDpl-WOX13 and ESExE-WOX13 motifs, respectively. Hence, the similarity between the YFdPMWOX10 OG and YxDpl-WOX13 motifs suggested a common origin. Partial protein sequences from Brassica oleracea and Raphanus raphanistrum subsp. raphanistrum (protein sequence derived from the two ESTs, gb|EX762111| and gb|EX766460|) confirmed the specificity of the motifs to Brassicaceae. Thus, the AtWOX14 containing branch appears to be Brassicaceae specific since: 1) all the sequences containing the two AtWOX10, 14 motifs are from Brassicaceae and 2) no species other than those belonging to the Brassicaceae displayed any of these two motifs.

The Gymnosperm and moss branch contained proteins that shared the ESExE-WOX13 OG motif with the Angiosperm WOX13 proteins, located at the same position relative to the HD. They also shared two other motifs specific to this branch: 1) the LxxGQ-gymnosperm/mossWOX OG motif that was at the same position as the YxDpl-WOX13 OG or YFdPM-WOX10 OG motifs and 2) a supplementary motif in the $\mathrm{C}$-terminal region of the proteins.

\section{Functional WOXI3 OG genes in the three model plants} We investigated the expression profiles of the WOX13 OG genes within 3 plant model species namely $O$. tauri, $P$. patens and $A$. thaliana because of their position within the green lineage. O. tauri is a highly proliferating unicellular alga that has no known differentiated form. In addition to the WOX gene, we also identified a single copy of a KNOX-like gene (Ot04g04460) within the O. tauri genome. In Angiosperms, members of the class-1 KNOX subfamily act to prevent cell differentiation within the SAM [26]. Interestingly, the OtKNOX protein showed the characteristics of both the class- 1 and class- 2 KNOX conserved domains, suggesting that $O$. tauri had diverged from a common ancestor before the diversification of the KNOX gene into 2 classes.

In order to monitor the expression of WOX and KNOX genes during the progression of the cell cycle, we partially light-synchronized a cell suspension of $O$. tauri as described before in [27]. RT-PCR and quantitative PCR analyses of Cyclin B and Histone 4 genes showed a peak of expression at $14 \mathrm{~h}$, confirming the overlapping of the G2/ $\mathrm{M}$ and $\mathrm{S}$ subpopulations (Figure $3 \mathrm{~A}$ and see additional file 4: Figure 1). The OtRH4 (Ot07g03220), and OtRH21 (Ot10g01950) genes, respectively the orthologues of the Arabidopsis DEAD-box RNA-Helicase 4 and 21 [28] were used as controls of constitutively expressed genes. They did not show any significant variation, and they were not cell cycle regulated (Figure 3A). More importantly, the WOX and the KNOX genes were found to be constitutively expressed and not cell cycle regulated (Figure 3A), with a higher expression level for WOX compared to KNOX (see additional file 4: Figure 1).

In $P$. patens, the initial gametophytic phase consists of an array of filamentous tubes that are formed after cell initials are produced from the main filament. Further development proceeds by the formation of buds which form the initial meristem from which originates the leafy adult gametophyte [29]. These processes rely on a tight control of cellular differentiation throughout the gametophyte development. An analysis of WOX expression in P. patens was carried out on 4-, 10- and 14-day-old plants corresponding respectively to the protonema, the bud and the gametophore stages under our growth conditions (data not shown). RT-PCR analyses showed that PpaWOX01 and PpaWOX02 were expressed throughout gametophyte development whereas PpaWOX03 transcripts were not detected (Figure 3B), thus supporting the hypothesis that PpaWOX3 could be a pseudogene.

An exhaustive data mining analysis of $A$. thaliana expression databases [30-33] indicated that, both AtWOX13 and AtWOX14 transcripts are present at different developmental stages $i$. e., embryo development, plantlet stage, root formation, bolting stage, in flowers and in response to stress (see discussion). However, no AtWOX10 transcripts were present in any of the databases analyzed. In addition, RT-PCR analyses of seedlings, even with a high PCR cycle number, did not allow us to detect the AtWOX10 mRNA 


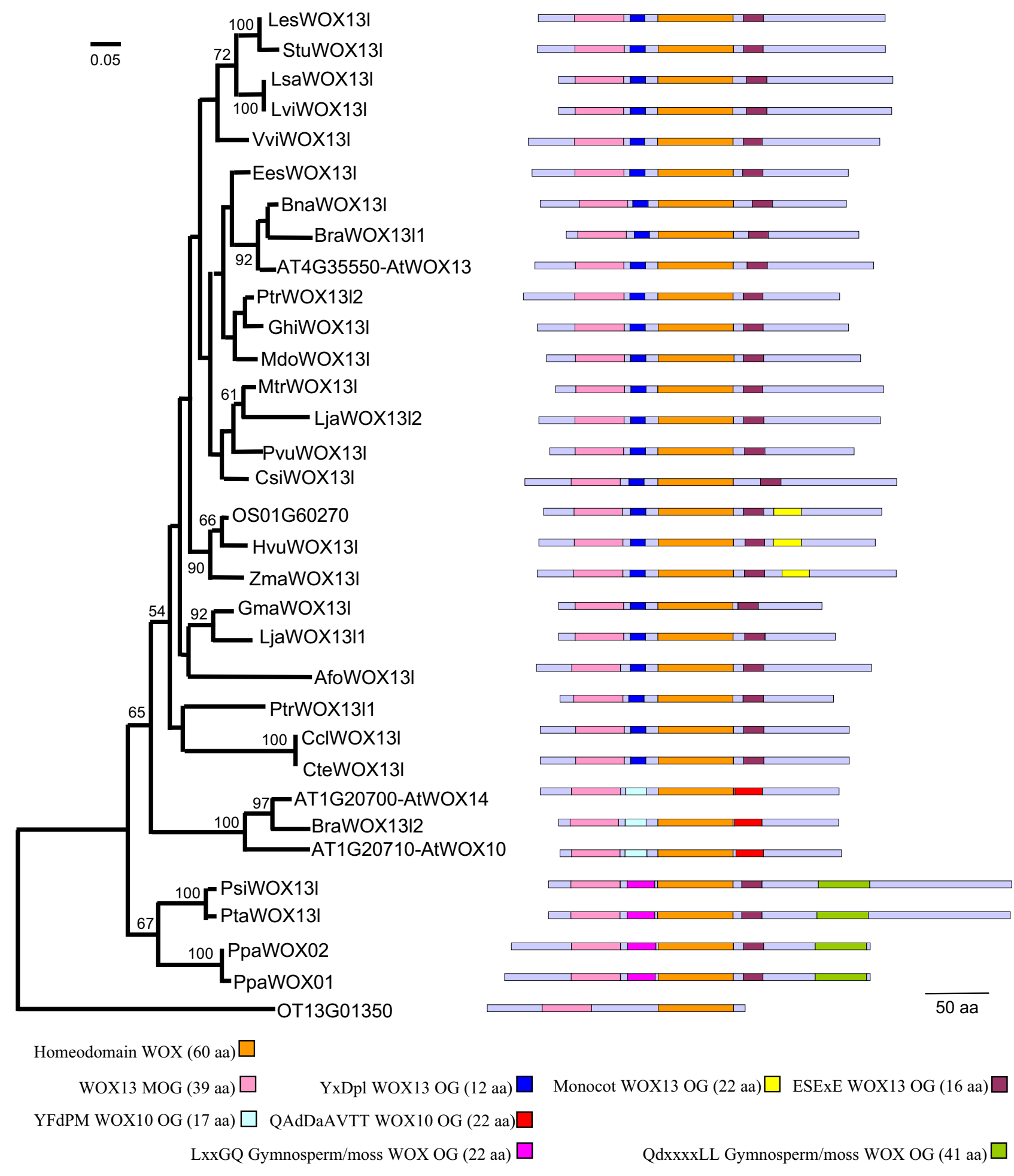

Figure 2 (see legend on next page) 
Figure 2 (see previous page)

The WOX 3 Orthology Group. The different elements of the figure are described from the left to the right. I) The neighbor-joining tree of the WOX proteins from the WOXI3 Orthology Group (see material and methods). The tree topology, the significance of the nodes, and the bootstrap values displayed are the same as in Figure I. The scale at the top left corner indicates 0.05 substitutions per site. 2) The motif composition of each protein is denoted by colored boxes on a bar representing the length of the protein and aligned on the WOX homeodomain (displayed in orange). Species names, in alphabetic order, are: Afo: Aquilegia formosa; AT: Arabidopsis thaliana; Bna: Brassica napus; Bra: Brassica rapa; Ccl: Citrus clementina; Csi: Citrus sinensis; Cte: Citrus temple; Ees: Euphorbia esula; Ghi: Gossypium hirsutum; Gma: Glycine max; Gra: Gossypium raimondii; Han: Helianthus annuus; Hvu: Hordeum vulgare; Les: Lycopersicon esculentum; Lja: Lotus japonicus; Lsa: Lactuca sativa; Lvi: Lactuca virosa; Mdo: Malus domestica; Mtr: Medicago truncatula; OS: Oryza sativa; OT: Ostreococcus tauri; Ppa: Physcomitrella patens; Psi: Picea sitchensis; Pta: Pinus taeda; Ptr: Populus tremula $\times$ Populus tremuloides; Pvu: Phaseolus vulgaris; Stu: Solanum tuberosum; Vvi: Vitis vinifera; Zma: Zea mays. The color code of the different motifs is described at the bottom of the figure.

(Figure 3C). These results suggested that the recently duplicated AtWOX10 gene, found only in the A. thaliana genome, might be a pseudogene. Based on these observations, this gene was not further characterized. We conclude that in the three plant model genomes studied at least one copy of the WOX13OG genes is expressed during development.

\section{AtWOXI3 shows a dynamic expression profile during A. thaliana development}

Compared to other WOX proteins, the function of the AtWOX13 and 14 proteins have received little attention to date (see review [22]). In order to carry out a more detailed analysis of the WOX13 OG genes in A. thaliana, we cloned the gene coding for the GUS reporter under the control of the AtWOX13 and AtWOX14 upstream regulatory sequences. Based on our data mining analyses (see above), we first analyzed the GUS expression during root development. AtWOX13 promoter activity was detected during lateral root formation (Figure 4A, C, F and 4I). To validate the specificity of these data, in situ hybridization experiments were performed using respectively AtWOX13 (Figure 4B, D, G and 4K), GUS (Figure 4J) and CUC2 as probes (Figure 4E and $4 \mathrm{H}$ ). The signal obtained using the AtWOX13 and GUS probes matched perfectly with the above GUS staining, whereas the CUC2 probe, as expected, did not give any specific signal. Hence, the GUS staining obtained reflects stable tissue specific mRNA expression and revealed that the AtWOX13 promoter sequence, used to drive the reporter gene GUS expression was sufficient to mimic the tissue specific localization of the endogenous AtWOX13 mRNA.

AtWOX13 expression was detected at the early stage of the lateral root (LR) formation of 10-days-old seedlings and it persisted during LR emergence (Figure 4A-D). The GUS staining was progressively restrained to the distal meristem (Figure 4F, and 4G) and it strongly accumulated in both the columella and the adjacent lateral root cap cells at a later developmental stage (Figure 4I-K). In mature LR or primary roots of 10-day-old seedlings no staining was detected within the root tip.

The AtWOX13 expression pattern was also investigated using the AtWOX13::GUS lines, during plant development. In 5-day-old seedlings, the promoter activity was strong in both cotyledon and root vasculatures, the pericycle and in the stem cells of the primary root meristem (Figure $4 \mathrm{M}$ and $4 \mathrm{~N}$ ). No GUS staining was detected in the division zone of the root. In the vegetative apex of 10 dayold seedlings, a strong staining was observed in the leaf primordia (Figure 4O). In the flower, the AtWOX13 promoter was active in the vasculature, the stigma (Figure $4 \mathrm{P}$ ) and in the gynoecium at the flower stage 13/14 (Figure $4 \mathrm{Q}$, and $4 \mathrm{R}$ ). At this developmental stage, when fertilization was ongoing and pollen grains were germinating at the tip of the gynoecium, ovules stained blue (Figure 4Q). Later on, the young embryos remained stained (Figure $4 \mathrm{R}$ ), but no staining was observed in mature siliques indicating that the AtWOX13 promoter was no longer active (Figure 4S), as observed before in all the other mature organs. During germination, soon after seed imbibition, GUS staining was also seen within the vasculature and the root tip, as observed in young seedlings (data not shown). Hence, AtWOX13 expression was restricted to differentiating organs and therefore probably regulated by developmental signals.

\section{AtWOX 14 promoter specificity differs from that of AtWOX 3}

Compared to the AtWOX13 expression patterns, the activity of AtWOX14 was more restrictive during lateral root formation. As for AtWOX13, the AtWOX14 promoter activity, revealed by GUS staining, was detected in the LR primordia, but decreased progressively as the LR emerged becoming restricted in fine to the vasculature up to the LR junction (Figure 5A-C). The AtWOX13::GUS gene continued to be expressed for a longer time (compare Figure $5 \mathrm{~B}$ with Figure 4C). In 5-day-old seedlings, AtWOX14 driven GUS activity was only found in the vascular system and in the pericycle of the primary root (Figure 5D). In the LR of 
14-day-old seedlings (Figure 5E), GUS staining was again observed in the vasculature and the pericycle with AtWOX14 expression being progressively induced in the pericycle and in the vasculature as the LR developed. Compared to the activity of the AtWOX13 promoter,

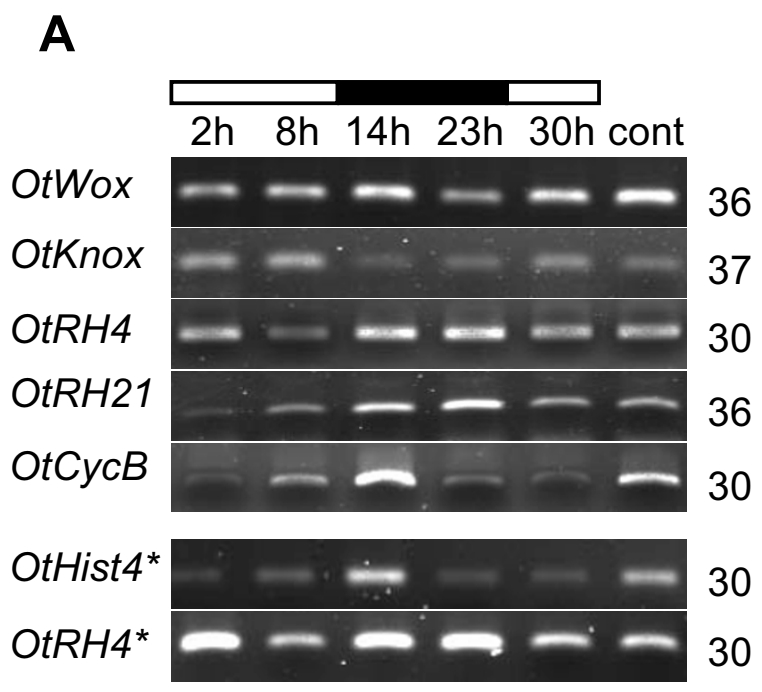

B
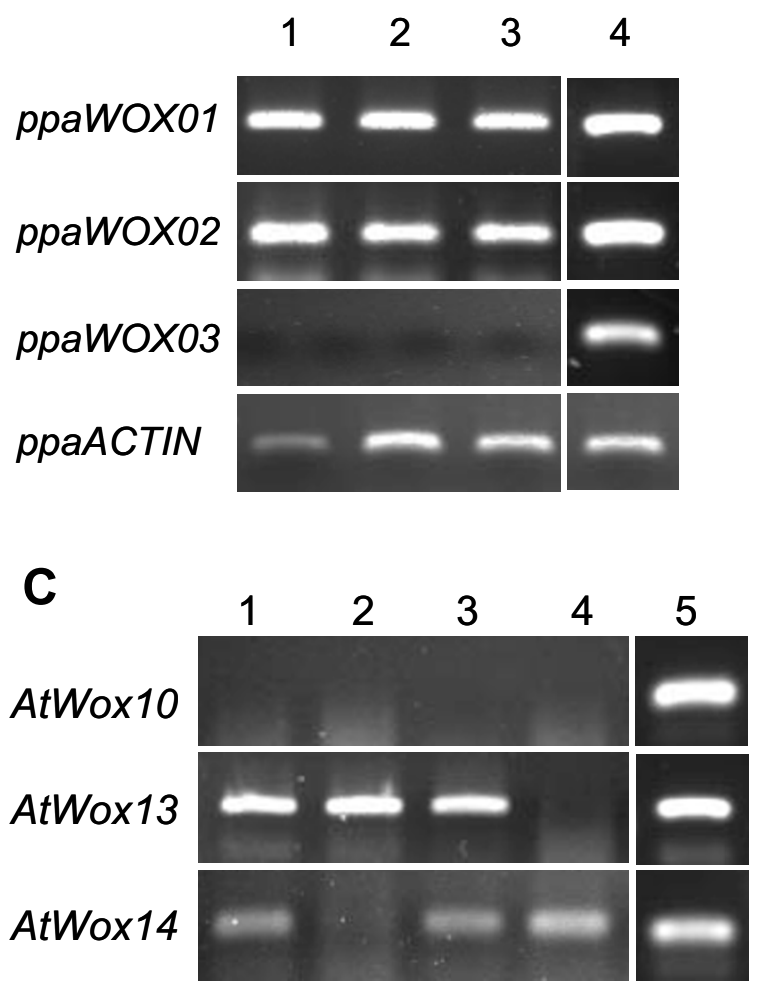

Figure 3

\section{Figure 3}

WOX 13 OG gene expression in O. tauri, $P$. patens and $A$. thaliana. (A) RT-PCR analysis of WOX gene expression in Ostreococcus tauri. $12 \mathrm{~h}$ light/dark synchronized or continuous light (cont) cell cultures of I0-day-old were used. Gene name (left side), number of PCR cycles done (right side), time of sampling (top side), light (blank bar) and dark (black bar) periods. The genes OtRH4 and OtRH2I were used as constitutive expression controls. RT were done using oligodT primers except in $\left(^{*}\right)$ where random primers were used (B) RT-PCR analysis of WOX gene expression during Physcomitrella patens development: (I) 4-day-old, (2) I0-day-old, (3) I4-day-old, (4) genomic DNA control. (C) RT-PCR analysis of the expression of WOXI3 OG genes carried out on 7-day old seedlings of different Arabidopsis thaliana lines: (I) Wild type No ecotype, (2) the pst I 3645 AtWOX 14 mutant line, (3) Wild type WS ecotype, (4) the I93G I0 AtWOXI 3 mutant line, (5) Genomic DNA control.

AtWOX14::GUS expression stopped at a longer distance further up from the root tip (Figure 5E, black arrow), exactly at the position where the youngest LR primordia were initiated (see arrow in Figure 5F).

During flower development, AtWOX14::GUS expression was detected in the stamen at floral stage 11/12 (Figure $5 \mathrm{G}-\mathrm{I})$, when mitotic divisions of microspores are known to occur [34]. The staining decreased as the stamen matured, showing that the function of WOX14 is linked to the early stages of organ and tissue development. Hence, as for AtWOX13, AtWOX14 promoter activity also seems to be regulated by developmental signals. The gene expression profile reported in the gene atlas [35] and the tissues and organs affected by the mutation of AtWOX14 (see below) agree with the expression profile described above.

\section{An AtWOXI3 gene truncated downstream of the} homeodomain neither alters root nor flower development The only available wox13 mutant line, identified in GABIKat [36] and numbered 193G10, showed a T-DNA insertion upstream of the sequence coding for the characteristic WFQN motif located at the end of the WOX homeodomain (Figure 6A and see additional file 4: Figure 2A). RTPCR experiments carried out using polyA+-RNA, prepared from the wox $13193 \mathrm{G} 10$ line, and primers matching each side of the insertion site, did not yield any PCR product (see Figure 3C). Because of the position of the insertion at the end of the WOX homeodomain, we also carried out an expression analysis using another couple of primers matching the 5'UTR of the AtWOX13 mRNA and the left border of the T-DNA. The latter experiment led to the amplification of a 500 bp PCR product, the sequence of which coded for a putative chimeric protein of 148 resi- 

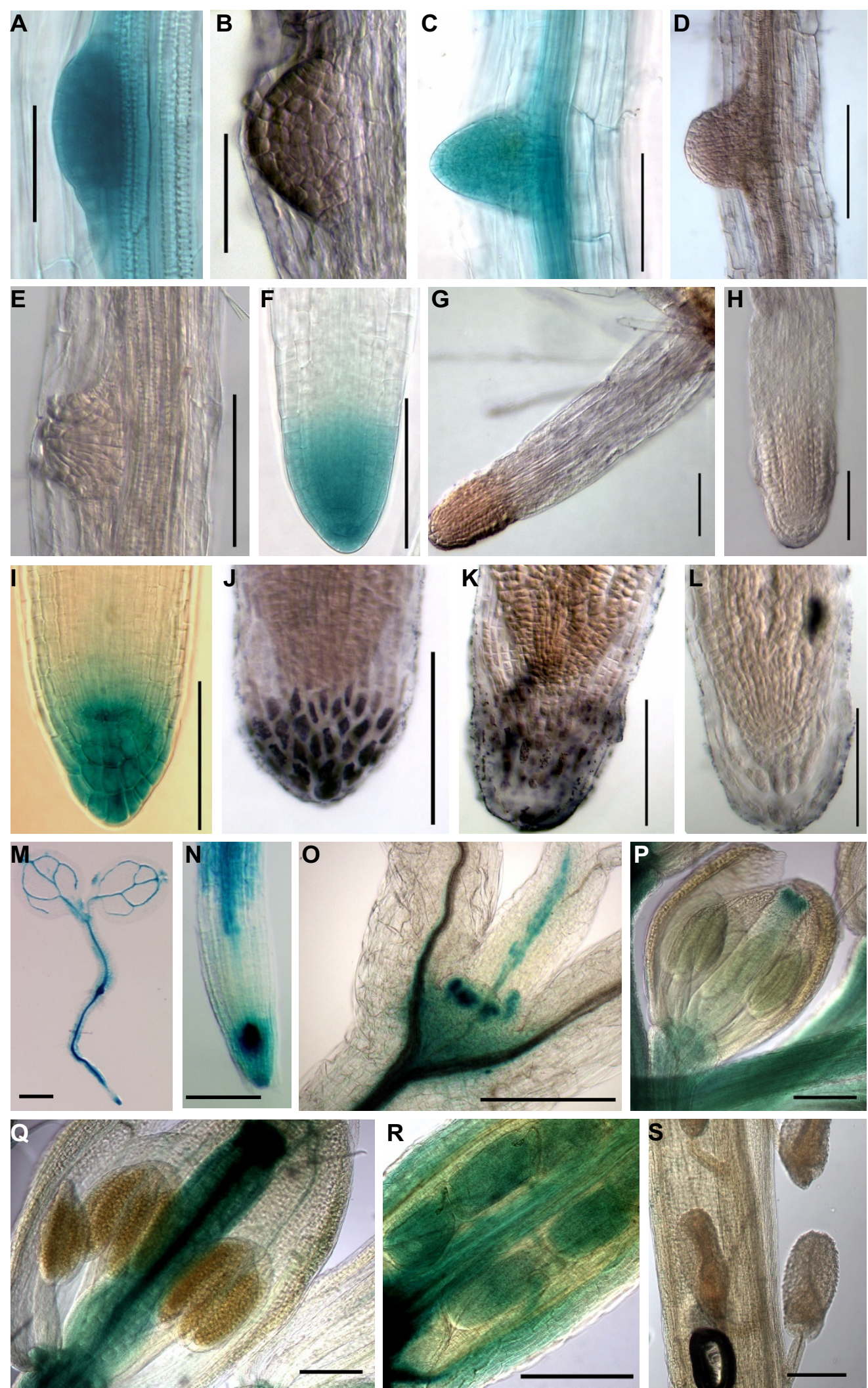

Figure 4 (see legend on next page) 
Figure 4 (see previous page)

AtWOXI3 gene expression during plant development. (A to $\mathbf{L}$ ) Expression profile during the initiation and development of lateral roots obtained with I0-day-old AtWOXI3::GUS seedlings. Independent lines were tested. (A, C, F and I) GUS staining. (B, D, and K) In situ hybridization with an AtWOXI 3 antisense probe. (J) In situ hybridization with a GUS antisense probe used as a positive control. (E, $\mathrm{H}$ and $\mathrm{L}$ ) In situ hybridization with a CUC2 antisense probe used as a negative control. ( $M$ to S) Expression profile at different plant developmental stages with independent AtWOXI3::GUS lines. (M) 5-day-old seedling with staining in the leaf, the hypocotyl, the root vasculature, the pericycle and the primary root tip. (N) Magnification of a 5day-old seedling primary root tip showing high staining in the stem cell of the proximal meristem. (O) Expression within the shoot apex of 10-day-old seedlings. Note the staining in the leaf primordia. (P to S) Expression within the flower at different developmental stages where staining is seen in the stigmata and the vasculature $(P)$ in the gynoecium $(Q)$ at the floral stage I2I 3 where the ovules $(Q)$ are stained. (R) Young embryo, (S) Mature silique. Scale bars = I00 $\mu \mathrm{m}$ except for A, B, R and S (= 50 $\mu \mathrm{m})$ and $M(=500 \mu \mathrm{m})$.

dues composed of the 143 amino acids of the AtWOX13 $\mathrm{N}$-terminal region plus an additional 5 amino acids specified by the T-DNA sequence.

Based on the expression profiles obtained using the GUS reporter gene constructs, an analysis of the primary root growth of the mutant lines was carried out after seed germination. Surprisingly, the above WOX13 mutation neither affected primary root growth (Figure 6C), nor vegetative and flower development. The only phenotype appeared to be a much earlier floral transition (Figure 6E).

\section{A WOX 4 knock-out mutant modifies lateral root and stamen development of $\mathrm{A}$. thaliana}

Conversely to the wox13 mutant, a wox14 insertion mutant line (i.e., pst13645) with the entire WOX homeodomain deleted (Figure 6B and see additional file 4: Figure $2 \mathrm{~B}$ ) displayed developmental defects in all the organs where GUS staining was observed. The primary root growth was retarded (Figure 6D) and after flowering, the pst13645 insertion line showed partial sterility (Figure $6 \mathrm{M})$, with aborted and shorter siliques compared to the wild type (Figure $6 \mathrm{~N}$ ). The floral transition was also altered, however the phenotype observed was that of a late flowering mutant (Figure 6F).

The wox14 mutant was further characterized during plant development. Whereas in the wild type Nossen a fully developed network of LR was observed (Figure 6G, H), the LR formation in wox14 was strongly inhibited (Figure 6I, J). The pst13645 line displayed extra adventitious roots (see Figure 6K, L) whereas the wild type plants developed only one or two. At the reproductive stage, observations after tissue clearing showed that the shorter siliques of wox14 contained many ovules that did not develop into seeds (Figure 6P), compared to the wild type (Figure 6O). Since the AtWOX14 promoter was only active in the developing stamen, pollen grain viability was checked using Alexander staining. As shown in Figure 6Q and 6R, the anthers of both the wild type and the wox14 mutant contained viable pollen grains when compared to the sterile dmc1 RNAi line [37]. However, stamen development was severely affected in the wox14 mutant. In open flowers of the wild type plants, the stamens were longer than the gynoecium allowing complete fertilization of the ovule (Figure 6T). In contrast, in the mutant line, the stamen did not fully develop and remained shorter than the gynoecium thereby preventing efficient fertilization and causing ovule abortion. In some wox 14 flowers, the phenotype was even more severe, with aborted stamens that developed to only half the size of the gynoecium (Figure 6V). These data suggested that AtWOX14 prevents premature organ and tissue differentiation at the floral stage.

\section{Discussion}

To improve previously described and not completely congruent WOX phylogenies $[14,16,38]$ we performed multi alignments on protein sequences (60-61 amino acids) longer than the usually defined HD (41-42 amino acids). Furthermore, we used gene or protein features other than the HD sequence to support the three OGs exhibited by our phylogenic trees. Even if the WOX gene phylogeny remains difficult to reconcile with species phylogeny, we are confident about the gene distribution between the three main OGs. Only one WOX gene was detected in the basal unicellular and photosynthetic organism $O$. tauri and three WOX genes were identified in moss $P$. patens, including at least two functional paralogues. The WOX13 OG is the clade containing the most conserved WOX genes as it is the only clade that contains the O. tauri, the $P$. patens and probably the eight $S$. moellendorffii genes. It also showed a recent and specific expansion in Brassicaceae. These observations point out the interest in the study of the WOX13 OG with respect to its biological specificity and gene family evolution.

\section{WOXI4 orthologues are only observed in Brassicaceae species}

We detected AtWOX13 and AtWOX14 mRNAs at different stages of plant development, in plantlets, roots, flowers and developing embryos. These expression profiles were in accordance with those described in a compendium of 

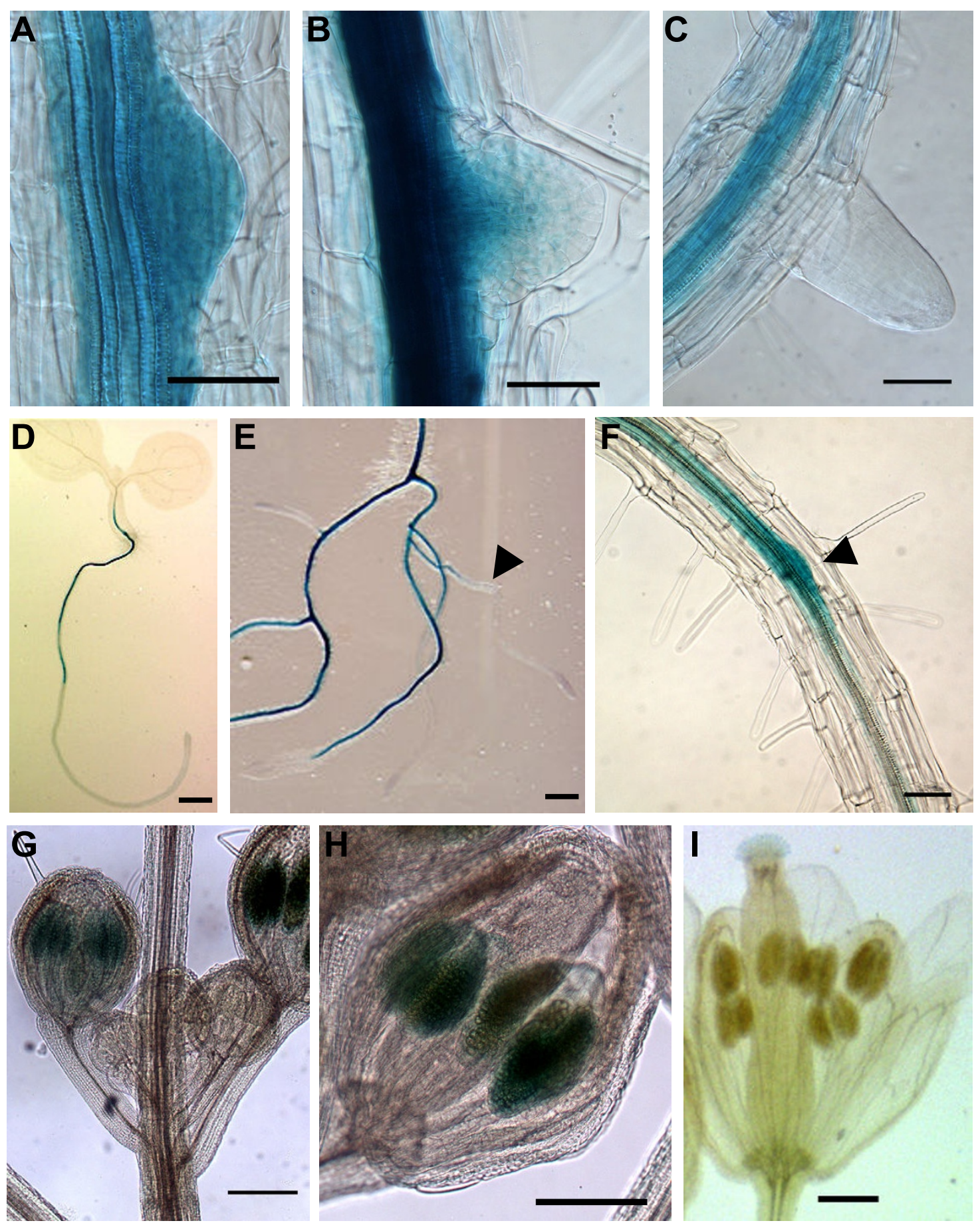

\section{Figure 5}

AtWOX 14 gene expression during plant development. (A to C) Expression profile during the initiation and development of lateral roots obtained with I4-day-old AtWOX I 4::GUS seedlings. Independent lines were tested. (D) 5-day-old seedling showing staining in the lower part of the hypocotyl and the upper part of the primary root vasculature. (E) Staining profile in primary and lateral roots of I4-day-old seedlings with expression in the upper root vasculature and pericycle. (F) Magnification at the edge of the GUS coloration showing the presence of the youngest LR initial (black arrowhead). (G to I) Expression within the flower showing strong staining in the anther only at floral stage II-I2 (G and H) before opening. Note the absence of staining in the mature anther (I). Scale bars $=100 \mu \mathrm{m}$ except for $\mathrm{C}(=200 \mu \mathrm{m})$ and $\mathrm{D}, \mathrm{E}(=500 \mu \mathrm{m})$. 

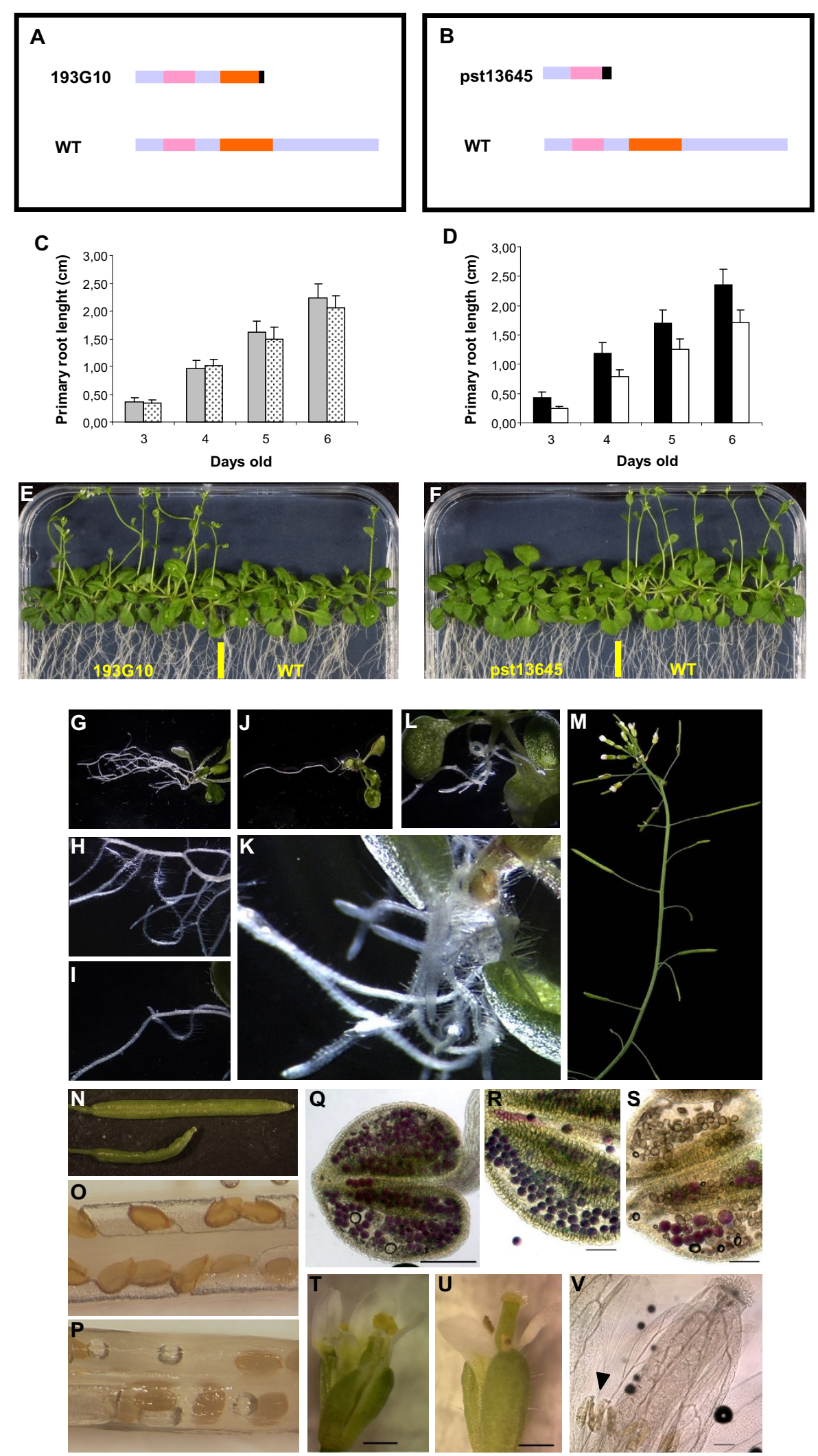

Figure 6 (see legend on next page) 
Figure 6 (see previous page)

The effects of the WOXI3 OG mutations on Arabidopsis thaliana development. (A) Diagram of the AtWOXI3 protein of the wox 13 line $193 \mathrm{GI} 0$ and the wild type plants (WT). (B) Diagram of the AtWOXI4 protein of the wox 14 line pst 3645 and the wild type plants (WT). Legends: WOXI3MOG (pink), HD (orange)(C and D) Comparative primary root growth of mutant and wild type seedlings during the first 6 days of development. In (C), wild type WS ecotype (grey bar) and wox 13 line I93GI0 (dot bar). In (D), wild type No ecotype (black bar) and wox I 4 line pst I 3645 (white bar). (E) Floral transition of wox 13 and WS plants after a long day period of 4 weeks.(F) Floral transition of wox 14 and Nossen plants after a long day period of 4 weeks. ( $G$ to $\mathbf{K})$ Root development of the wild type plant $(\mathrm{G}$ and $\mathrm{H})$ compared to the wox I 4 line pst I 3645 (I to $K$ ) that overproduces adventitious roots. ( $\mathbf{M}$ to $\mathbf{P})$ Flower development of wox $/ 4$ line pst 13645 inflorescences that show partial sterility (M). Wild type (upper silique in $N$ and $O$ ) and wox / 4 semi-sterile silique (lower siliques in $N$ and $P$ ). (N and $P$ ) Pictures obtained after clearing. ( $Q$ to $\mathbf{S})$ Pollen viability tests with Alexander staining. (Q) Wild type, (R) wox/4 pollen. (S) dmc I/RNAi sterile mutant. (T to V). Mature flower development in wild type plants (T) and the wox I 4 line (U and V). (V) Picture after clearing. Scale bars: $\mathrm{Q}, \mathrm{V}(=\mathrm{I} 00 \mu \mathrm{m}), \mathrm{R}, \mathrm{S}(=50 \mu \mathrm{m})$ and $\mathrm{T}, \mathrm{U}(=500 \mu \mathrm{m})$.

A. thaliana expression databases for the WOX13 OG genes [30-33]. For instance, in the gene expression map of the Arabidopsis root [39], the AtWOX13 mRNA profile shows a localized expression domain (LED) specific to the stele in the elongation zone of the mature primary root as observed in our study. These databases also revealed that the expression levels of both AtWOX14 and AtWOX13 were low compared to other WOX genes, suggesting either a tight control of their expression or a cell specific expression pattern. This has been confirmed by the expression data described in this paper.

The AtWOX14 branch within the WOX13 OG, has previously been suggested to be unique to A. thaliana [14]. Our study based on more species indicates that this branch also contains sequences from other Brassicaceae. Interestingly, differences in the expression profiles between AtWOX14 and AtWOX13 indicate that their 5' regulatory elements have diverged since gene duplication. In line with this conclusion, our data mining analyses showed that AtWOX13 expression, but not that of AtWOX14, was modulated in response to biotic and abiotic stresses. Hence, the AtWOX14 promoter appears to have diverged to presumably support specific developmental mechanisms found so far only in Brassicaceae. Analysis of the expression profile of a non Brassicaceae WOX13 gene should give us a better understanding of the evolutionary mechanisms giving rise to this particular branch.

\section{WOXI3 OG genes affect organogenesis and floral transition}

The control of cell proliferation and differentiation during organ development are essential processes that require genes implicated in cell signaling, cell identity and cell cycle transitions. In higher plants, both the SAM and the root pericycle cells serve as initiation sites of organogenesis $[40,41]$. In this paper, we show that AtWOX13 and AtWOX14 are active in organ primordia and during early tissue differentiation. In the transcriptomic database CATdb [30], in which only the AtWOX13 probe is availa- ble, 5 out of 62 experiments associate AtWOX13 gene expression with cell proliferation and growth. Interestingly, the transcriptome profiling experiments, using protoplast cultures [42], showed that the AtWOX13 mRNA level followed an expression profile similar to that of the proliferating cell nuclear antigen 1 gene (see additional file 4: Figure 3A), a key regulator of DNA metabolism and cell cycle progression [43]. Conversely, the homeobox gene $A T H B 7$, a marker of cell differentiation and elongation [44], strongly decreased in the same protoplast culture. Compared to the cell cycle gene (see additional file 4: Figure 3B), AtWOX13 expression levels were maximal when the cells entered cell division. AtWOX13 expression was also up-regulated in the tor mutant or after a treatment with the IP3K inhibitor, wortmannin. The TOR protein and IP3K are regulators of cell division and growth. TOR has only been found in non-differentiated and rapidly dividing meristematic cells $[45,46]$. However, the dynamic expression of AtWOX13 during LR development described in this paper does not entirely overlap with the cell division zone, as AtWOX13 promoter activity became more restricted to the vasculature of the root.

Our data also showed that mutations of the AtWOX13 and 14 genes affected the floral transition. An increase of cell division in all the vegetative SAM was proposed to be a prerequisite for morphological changes at the floral transition in plants $[47,48]$. Furthermore, in A. thaliana stem axis growth is the result of cell elongation that occurs only in the rib zone and not in the upper part of the SAM [49]. Hence, the late flowering phenotype observed in the wox14 mutant might reflect a default in the coordination of cell division and elongation in the transitional SAM. In contrast, the wox13 mutant line showed a surprising early floral transition. However, a chimeric WOX13 mRNA coding for a putative protein containing the conserved domains was expressed in the wox13 193G10 line. We suggest that this line might act as a dominant negative mutant that induces early floral transition by activating precocious cell proliferation in the SAM. Alternatively, we 
cannot exclude that other WOX family members could fulfill the function of the AtWOX13 protein, leading to a weak phenotype in the Arabidopsis mutant background. To date, the mechanism underlying gene regulation by the homeobox gene family is complex and remains largely unclear [50]. A monitoring of the promoter activity during the floral transition and an analysis of a mutant line with an insertion upstream of the HD or an RNAi strategy should give us better insights into the function of the WOX13 protein.

Finally, we found that the wox14 null mutant showed root growth delay and early anther maturation. Interestingly, a similar phenotype was also observed in anthers when cyclin-dependent kinase inhibitors were ectopically expressed in plants [51-53]. Hence, it is tempting to speculate that the AtWOX14 protein is involved in the negative control of cell differentiation to allow for correct development.

In a similar way, the expression profile observed during the gametophytic development of $P$. patens is consistent with a putative function in cell differentiation control during the filamentous growth and the bud formation although in situ hybridization experiment are needed to validate this hypothesis. Last, the constitutive expression of the only WOX gene in O. tauri may account for its maintenance in an undifferentiated state. Future use of $O$. tauri and $P$. patens as biological models and ongoing complementation experiments of mutants using either full length or truncated proteins should allow us to obtain a better understanding of the functional importance of the WOX13 OG genes.

\section{Conclusion}

Our data provide evidence in favor of the WOX13 OG as the clade containing the most conserved WOX genes. The preferential presence of amino acid motifs fully supports the separation of the different gene groups in the phylogenetic trees. However, the latter is not consistent with the green plant phylogeny [21]. In this study, we linked the function of the WOX13OG Arabidopsis members to organ initiation and development, most likely by preventing premature differentiation as shown for other WOX proteins. The data also suggest that the WOX family has diverged both at the transcriptional and protein level to generate new features that control cell identity in specific domains of land plants. In line with such evolutionary events, the expression profile and mutant phenotype of the AtWOX14 gene suggest that the Brassicaceae might have developed specific mechanisms to control floral transition and pollen formation when compared to other plants.

\section{Methods \\ Identification of WOX genes}

The identification of WOX genes was undertaken using model plants for which the complete genome was available, i.e., A. thaliana (a core eudicot), O. sativa (a monocot), P. patens (a moss) and O. tauri (a green alga). The WOX family was defined in A. thaliana and O. sativa genomes after a BLASTp [54] search in the FLAGdb++ database [55] using AtWUS (AT2G17950). AtWUS-like sequences were identified in the $P$. patens whole genome shotgun database ftp://ftp.ncbi.nih.gov/pub/TraceDB/ physcomitrella patens/, the O. tauri [56] and the O. lucimarinus [57] genomes after a BLASTp using the HD amino acid sequence. The limits of the sub-families were defined following the drop-in-Blast score method [58,59]. The structures of the identified genes were verified using all of the corresponding transcripts and when necessary the gene was reannotated. There was only one WOX gene, (OT13G01350), within the O. tauri and the O. lucimarinus genomes. Gene and protein sequences of the WOX families from completely sequenced genomes of model species are given (see additional file 3: Table 1). The positions of introns were obtained from nucleotide sequence alignments derived from the protein alignments. Only introns inserted into genomic regions coding for sufficiently similar protein sequences were used to study the conservation of intron insertion sites.

The proteome of S. moellendorffii was downloaded from the JGI ftp site $\mathrm{ftp} / / / \mathrm{ftp}$.jgi-psf.org/pub/JGI data/ Selaginella moellendorffii/. Using the WOX-HD HMM (see additional file 3: Table 2), we found 8 WOX sequences, but only 3 were apparently full-length. Only the complete SmWOX sequences were used for the phylogeny reconstruction of the WOX family.

\section{Distance trees and phylogeny reconstruction of the WOX family}

Neighbor-Joining (NJ) [60], Maximum likelihood (ML) [61] trees, both based on Jones-Taylor-Thornton matrix [62], and Parsimony [63] trees were constructed using PHYLIP [64]. To assess support for the calculated relationships, 1000 bootstrap samples were generated [65]. To select appropriate outgroups for the WOX model plant phylogeny, we first established a tree using all of the HD proteins from A. thaliana and 3 outgroups (i.e., At3G03260, At4G00730, At4G17710) were chosen from the three clades closest to the WOX clade. Trees were computed from 60 to 61 amino acid sequences containing the HD of the WOX proteins from the five plant model genomes, O. tauri, P. patens, S. moellendorffii (only the 3 available full-length proteins were used), A. thaliana and $O$. sativa. The clustering of the WOX proteins in three different OGs obtained from the genome models were consistent using both NJ (Figure 1) and ML (see additional 
file 1: Figure 1) methods and the 3 above outgroups either independently or together (data not shown). We also built up phylogenetic tree from the same set of data using the Parsimony method. Different branching patterns were obtained depending upon which outgroup was used. With the multiple outgroup described above, $O$. tauri and $S$. moellendorffii were both independent branches at the base of the tree (see additional file 1: Figure 2).

The WOX13 OG trees has been established using the Neighbor-Joining [60] method based on Henikoffs evolutionary distances [66]. The trees obtained with the ML (see additional file 1: Figure 3) and Parsimony methods (see additional file 1: Figure 4) also gave the same main clusters of proteins.

\section{Analysis of WOX protein sequences}

In order to identify shared motifs among the protein sequences we used the MEME program, version 3.5.4 [67]. A gapped alignment using CLUSTALW was generated for each motif. Based on these multiple alignments a Hidden Markov Model (HMM) was built using HMMER 2.3.2 [20]. The HMM was used to search for motifs in the WOX sequences from the model genomes and in the whole proteome in GenBank NR (see additional file 3: Table 2). The sequence logos were made using Weblogo [68]. The specificity of the HMMs for the different WOX clades was tested using the two recently available Vitis vinifera genome sequences, PN40024 [69] and Pinot noir [70]. Using the WOX HD HMMs, an initial search in these two novel proteomes allowed us to specifically retrieve all members of the WOX gene family. A second search with the clade specific HMMs efficiently clustered the WOX genes into one of the three WOX clades (see additional file 3: Table 3). We found 13 different Vitis genes coding for WOX proteins, with 8 located in the WOX1 OG, 3 in the WOX13 OG, and 2 in the WOX8 OG. No V. vinifera proteins were located to the WOX14 clade.

\section{Plant material}

A naturally synchronized O. tauri strain OTTH0595 cell culture was used and the cell cycle analysed by flow cytometry as described by Farinas et al [27]. Using a $12 \mathrm{~h}$ light/dark condition, we obtained two subpopulations of synchronized cells at the light/dark transition, comprised of $12 \%$ cells in the $\mathrm{G} 2 / \mathrm{M}$ phase and the remaining cells in the $S$ phase of the cell-cycle. The moss $P$. patens was grown on BCD medium [71] overlaid with cellophane under 16 $\mathrm{h}$ light $/ 8 \mathrm{~h}$ dark cycle at $65 \%$ hygrometry and at $20^{\circ} \mathrm{C}$. The DS transposon tagged A. thaliana line wox14, i.e., pst13645, was obtained from the RIKEN BRC Japan. The T-DNA tagged A. thaliana line wox13, i.e., 193G10, was purchased from the INRA Versailles resource centre. The wox13 and wox14 mutant lines are in the Wassilewskija (Ws) and the Nossen (No) background, respectively. Wild type plants of both ecotypes were used as background controls.

\section{Root growth assays}

Sterile seeds were placed on a solid growth medium (MS salt supplemented with $20 \mathrm{~g} / \mathrm{L}$ sucrose and agar) at $4{ }^{\circ} \mathrm{C}$ for 2 days, and grown under $16 \mathrm{~h}$ light/ $8 \mathrm{~h}$ dark cycles $\left(20^{\circ} \mathrm{C}\right.$ during illumination, $18^{\circ} \mathrm{C}$ in the dark) at $65 \%$ hygrometry. Root length was measured from day 3 to day 6 using the ImageJ free software (NIH, USA) after scanning.

\section{Characterization of the mutant lines}

The pst13645 and 193G10 lines were screened using media containing either $30 \mathrm{mg} / \mathrm{L}$ hygromycin or $30 \mathrm{mg} / \mathrm{L}$ kanamycin, respectively. In order to verify the mapping of the insertion site, F2 or F3 plants were genotyped at the AtWOX13, At WOX14 loci. Specific genomic DNA primers and either T-DNA or the Ds-element border-specific primers were designed based on the annotated insertion site of each mutant line (see additional file 4: Table 1). The resulting PCR products were separated on a $2 \%$ agarose gel and their size was verified. $341 \mathrm{bp}$ and $163 \mathrm{bp}$ fragments were expected for the AtWOX13 wild type and mutated allele, respectively and $498 \mathrm{bp}$ and $216 \mathrm{bp}$ fragments for the AtWOX14 wild type and mutated allele, respectively. Moreover, the entire AtWOX14-AtWOX10 DNA region was genotyped to check for the absence of chromosome rearrangements of the duplicates and to confer that the phenotype was only due to the disruption of AtWOX14. Phenotypes were observed only in homozygous plants for each mutation and therefore they were used in all further experiments.

\section{Reverse transcriptase PCR}

Total RNA was extracted from the different plants using the Qiagen plant RNeasy extraction kit and DNAseI treated following the manufacturer's instructions. The RNA preparations were reverse transcribed using 1 to $5 \mu \mathrm{g}$ of RNA. RT-PCR experiments were performed in order to verify the expression of the different WOX and control genes (see additional file 4: Table 1) within the different plant model species and the transgenic Arabidopsis plants. Primer efficiency was tested using genomic DNA and eventual genomic contamination was always checked by PCR amplification of the RNA samples using the equivalent amount of CDNA used for the RT-PCR. Quantitative real-time RT-PCR was also performed on O. tauri mRNA as described previously [72] using a Roche Light Cycler ${ }^{\circledR}$ 480 with the SYBER ${ }^{\circ}$ green PCR mix from either Roche or Applied Biosystem. For each gene tested, PCR products from genomic DNA cloned into the $\mathrm{PGEMT}^{\circ}$ vector and the NotI digested empty plasmid were used as standards to quantify the corresponding RNA copy number. These raw 
copy numbers were then normalized to the amount of equivalent RNA used for the quantification.

\section{Construction of the WOX::GUS reporter genes}

To clone the AtWOX13 and AtWOX14 promoters, the entire intergenic regions upstream of the ATG initiators were PCR-amplified using gene specific primers containing either an $\mathrm{XbaI}$ or a BamHI restriction site (5'-TCTCGAGTGGAGCTTTTGCAGGTCTCT-3'; 5'-AGGATCCTCAG AATTTCGCTCAGAAGATTT-3') for AtWOX13 and containing either a NotI or a SpeI restriction site (5'GACAGCGGCCGCGGGGTTGTGAGTCCTATTGC-3'; 5'GACACTAGTTGAACAAGACAATGAGAAAGTGAA-3') for AtWOX14. DNA fragments (of $3.6 \mathrm{~kb}$ and $1 \mathrm{~kb}$ for the AtWOX13 and AtWOX14 promoters, respectively) were subcloned into the pGEMT vector (Promega) to give the prom WOX13 and prom WOX14 plasmids. For the WOX::GUS reporter constructs, prom WOX13 XhoI/ BamHI and prom WOX14 SpeI fragments were subcloned into a SalI/BamHI and an XbaI digested pPR97 binary vector, respectively [73]. Constructs were introduced into the Agrabacterium tumefaciens strain GV3101, then into Arabidopsis plants ecotype Ws as described in [74]. Transformed plants were selected by adding $30 \mathrm{mg} / \mathrm{L}$ kanamycin to the growth medium. Resistant plants were transferred to soil and grown in a growth chamber.

\section{Staining and observations}

Beta-glucuronidase (GUS) staining was performed for 24 $\mathrm{h}$ on $\mathrm{T} 1$ and $\mathrm{T} 2$ seedlings as previously described [75], except for the lateral roots where the incubation time was limited to $4 \mathrm{~h}$. After fixing and clearing, samples were mounted on slides in HCG (80\% Chloral hydrate, 10\% Glycerol, 10\% water) and observed under a Zeiss light microscope or a Zeiss binocular. Cytological observations of Alexander-stained anthers were carried out as previously described [76].

\section{In situ hybridization}

In situ hybridizations were conducted as previously described [77] using antisense probes. The AtWOX13 antisense probe was synthesized in vitro using a PCR product obtained with the following primers: (CCTGCAGATGATGGAATGGGATAATCAGC and TGTAATACGACTCACTATAGGGCACTGCTTATGACTGACTACCAAATCC). The CUC2 probe has been described previously [77] and the GUS probe was provided by the laboratory of P. Laufs (INRA Versailles).

\section{Authors' contributions}

The project was conducted by YD, MK and AL. The bioinformatics analyses were done by CTN, VT and AL; YD and GC carried out all the experimental works with the assistance of $\mathrm{H}$. Morin (in conducting the in situ hybridization) and $\mathrm{H}$. Moreau, the O. tauri experiments. YD, MK and AL wrote this manuscript; PL revised several versions of this manuscript. All authors read and commented on drafts of the manuscript and approved the final manuscript.

\section{Additional material}

\section{Additional file 1}

Phylogenetic trees. Figure 1 : Maximum-likelihood tree of the WOX proteins from the 5 completely sequenced genomes of the green lineage: Arabidopsis thaliana (AT prefix), Oryza sativa (OS prefix), Physcomitrella patens (Ppa prefix), Ostreococcus tauri (OT prefix) and the three full-length proteins of Selaginella moellendorffii (Sm prefix). The significance of each node was tested using 1000 bootstrap replicates. Only bootstrap values above $50 \%$ are shown. The scale, at the right of the tree, indicates 0.2 substitution per site. Figure 2 : Parsimony tree of the WOX proteins from the 5 completely sequenced genomes of the green lineage: Arabidopsis thaliana (AT prefix), Oryza sativa (OS prefix), Physcomitrella patens (Ppa prefix), Ostreococcus tauri (OT prefix) and the three full-length proteins of Selaginella moellendorffii (Sm prefix). The significance of each node was tested using 1000 bootstrap replicates. Only bootstrap values above $50 \%$ are shown. Figure 3 : Maximum-likelihood tree of the WOX proteins from the WOX13 Orthology Group. The significance of each node was tested using 1000 bootstrap replicates. Only bootstrap values above $50 \%$ are shown. The scale, at the right of the tree, indicates 0.1 substitution per site. Species names, in alphabetic order, are: Afo: Aquilegia formosa; AT: Arabidopsis thaliana; Bna: Brassica napus; $\mathrm{Bra}$ : Brassica rapa; $\mathrm{Ccl}$ : Citrus clementina; $\mathrm{Csi}$ : Citrus sinensis; Cte: Citrus temple; Ees: Euphorbia esula; Ghi: Gossypium hirsutum; Gma: Glycine max; Gra: Gossypium raimondii; Han:

Helianthus annuus; Hvu: Hordeum vulgare; Les: Lycopersicon esculentum; Lja: Lotus japonicus; Lsa: Lactuca sativa; Lvi: Lactuca virosa; Mdo: Malus domestica; Mtr: Medicago truncatula; OS: Oryza sativa; OT: Ostreococcus tauri; Ppa: Physcomitrella patens; Psi: Picea sitchensis; Pta: Pinus taeda; Ptr: Populus tremula $\times$ Populus tremuloides; Pvu: Phaseolus vulgaris; Stu: Solanum tuberosum; Vvi: Vitis vinifera; Zma: Zea mays. Figure 4 : Parsimony tree of the WOX proteins from the WOX13 Orthology Group. The significance of each node was tested using 1000 bootstrap replicates. Only bootstrap values above 50\% are shown. Species names, in alphabetic order, are: Afo: Aquilegia formosa; AT: Arabidopsis thaliana; Bna: Brassica napus; Bra: Brassica rapa; $C c l$ : Citrus clementina; $C s i$ : Citrus sinensis; Cte: Citrus temple; Ees: Euphorbia esula; Ghi: Gossypium hirsutum; Gma: Glycine max; Gra: Gossypium raimondii; Han: Helianthus annuus; Hvu: Hordeum vulgare; Les: Lycopersicon esculentum; Lja: Lotus japonicus; Lsa: Lactuca sativa; Lvi: Lactuca virosa; Mdo: Malus domestica; Mtr: Medicago truncatula; OS: Oryza sativa; OT: Ostreococcus tauri; Ppa: Physcomitrella patens; Psi: Picea sitchensis; Pta: Pinus taeda; Ptr: Populus tremula $\times$ Populus tremuloides; Pvu: Phaseolus vulgaris; Stu: Solanum tuberosum; Vvi: Vitis vinifera; Zma: Zea mays. Click here for file

[http://www.biomedcentral.com/content/supplementary/14712148-8-291-S1.pdf] 


\section{Additional file 2}

WOX gene structures and protein motifs. Figure 1 : The structure of WOX genes from model genomes. The gene structures from 4 model genomes (Arabidopsis thaliana, AT; Oryza sativa, OS; Physcomitrella patens, $\mathrm{Ppa}$; Ostreococcus tauri, OT) and three Selaginella moellendorffii $(\mathrm{Sm})$ genes are displayed following the order of the Neighbour-joining tree in Figure 1 and aligned on their translation start. Rectangles stand for exons while lines denote introns. Blue is for exonic regions in UTRs and light grey is for translated exons. Five different gene structures are given for PpaWOX02 due to the 5' UTR variants supported by transcripts. Figure 2: WOX proteins: Sequence alignments around the virtual intron insertion sites. For each $W O X O G, 15$ amino acid sequences both upstream and downstream introns are given. The intron insertion sites are indicated by stars. Numbers on the left of sequences give the intron length. Digits between stars stand for the phase of the intron insertion site. The intron phase is defined by the position of its insertion into a codon. An intron can be located between two codons (phase 0) or within a codon, lying either after the first or after the second base pair (phase 1 and phase 2 respectively). Boxes indicate conserved introns with the same color code than in Figure 1. Figure $3:$ Sequence logos of protein motifs in the WOX family. Each logo http://weblogo.berkeley.edu consists on stack of letters, one stack for each position in the sequence. The overall height of the stack indicates the sequence conservation at that position, while the letter's height within the stack indicates the relative information content of each amino acid at that position. The amino acid color code is dark blue for large polar, light blue for basic, black for small polar, green for nonpolar, orange for cysteine and purple for ambivalent amino acids. Figure 4 : WOX motifs: alignments for motifs without Logo. Motifs defined by less than 6 sequences cannot be shown as logo since the sequence information at each position is too weak. Thus multiple alignments performed with Clustalw with a majority consensus are shown here for theses motifs. Amino acid color code is the same than in Figure 2. Click here for file

[http://www.biomedcentral.com/content/supplementary/14712148-8-291-S2.pdf]

\section{Additional file 3}

WOX sequences and HMM motifs. Table 1: Gene and protein sequences of the WOX family in the model genomes A. thaliana, $\mathrm{O}$. sativa, P. patens and O. tauri. Table 2: Specificity and sensitivity of HMM for WOX motifs. Scores can give indications to distinguish potential family -or subfamily-members from false positive. A clear drop in the score could be detected in most cases, indicating that sequence below this threshold did not fulfill the family model as well as above [21]. HMMER bit scores were obtained for each motif by querying the HMM against three protein sequence sets: WOX13 OG (33 sequences), model genome WOX (32 sequences) and GenBank NR (Release 157). The score drops above 50 and indicating the motifs exhibiting the highest specificities with a given database are in bold. $a$ : no hit was found in the set. $b$ : no specific hit was found in the GenBank NR database. Note that Physcomitrella sequences from genome model WOX set are not present in GenBank NR. $c:$ best hits are not belonging to the WOX family and no score drop was found. Table 3: Vitis vinifera WOX protein prediction with HMM WOX motifs. HMM search scores above a drop give indication to assign novel WOX proteins to the different WOX OGs. AM(...) IDs are contigs from Pinot noir $\mathrm{V}$. vinifera while contig(...) IDs are from PN40024 V. vinifera. Dash indicates that no score was found above the drop. a: no hit was found with this HMM profile. $\left({ }^{*}\right): 17.4$ score is due to an undefined $C$ or T nucleotide at one position leading to a stop in the CDS. After fixing the nucleotide to $C$, the score is 27.7 .

Click here for file

[http://www.biomedcentral.com/content/supplementary/14712148-8-291-S3.pdf]

\section{Additional file 4}

Expression analyses. Figure 1 : Real-time PCR analysis of Ostreoccocus tauri gene expression in a 12-hour light/12-hour dark synchronized cell culture. Copy number of WOX, KNOX, Histone4 and CyclinB RNA was quantified using total RNA sampling from the starting of the light period $(O \mathrm{~h})$ or from continuous light grown cell culture. Figure 2 : Alignments of predicted sequences of AtWOX13 (A) and AtWOX14 (B) chimeric proteins with the full length ones. The homeodomain are indicated in bold and the amino acid changes at the end of the chimeric proteins indicated in lower case. Figure 3 : AtWOX13 expression is correlated with the induction of cell proliferation. Data mining analysis of the expression profiles of a set of genes in protoplast cul-

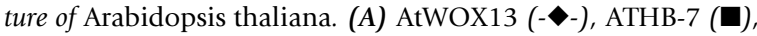
PCNA1 ( $\mathbf{\Delta})$; (B) Expression profile of a cluster of cell cycle genes including AT4G37490 (CYC1); AT1G44110 (CYCA1;1 (四); AT5G11300 (CYC3B); AT1G47210 (CYCA3;2); AT3G05330 (CDK); AT2G26760 (CYCB1;4); AT4G35620 (CYCB2;2); AT2G26430 (RCY1); AT2G31400 (ATPDNA Binding). Table 1: Primer sequences designed for expression analysis, genotyping ( $\mathrm{g}$ primers) and cDNA cloning (2 last row) using primer3 software.

Click here for file

[http://www.biomedcentral.com/content/supplementary/14712148-8-291-S4.pdf]

\section{Acknowledgements}

We thank Marta Kulis for useful help in promoter cloning, the Institute technical team for plant cultures, Roland Boyer (IBP, Orsay) for photographs, Evelyne Derelle, the training students and others that have contributed to this work supported by the CNRS and the Université Paris-Sud II. We are grateful to Michael Hodges and Michael Dubow for carefully reading and editing the manuscript.

\section{References}

I. Haecker A, Gross-Hardt R, Geiges B, Sarkar A, Breuninger H, Herrmann $M$, Laux T: Expression dynamics of WOX genes mark cell fate decisions during early embryonic patterning in Arabidopsis thaliana. Development 2004, I 3 I (3):657-668.

2. Nardmann J, Werr W: The Shoot Stem Cell Niche in Angiosperms: Expression Patterns of WUS Orthologues in Rice and Maize Imply Major Modifications in the Course of Mono- and Dicot Evolution. Mol Biol Evol 2006.

3. Gallois JL, Nora FR, Mizukami Y, Sablowski R: WUSCHEL induces shoot stem cell activity and developmental plasticity in the root meristem. Genes Dev 2004, I 8(4):375-380.

4. Park SO, Zheng Z, Oppenheimer DG, Hauser BA: The PRETTY FEW SEEDS2 gene encodes an Arabidopsis homeodomain protein that regulates ovule development. Development 2005, |32(4):84|-849.

5. Kamiya N, Nagasaki H, Morikami A, Sato Y, Matsuoka M: Isolation and characterization of a rice WUSCHEL-type homeobox gene that is specifically expressed in the central cells of a quiescent center in the root apical meristem. Plant J 2003, 35(4):429-44I.

6. Wurschum T, Gross-Hardt R, Laux T: APETALA2 regulates the stem cell niche in the Arabidopsis shoot meristem. Plant Cell 2006, I 8(2):295-307.

7. Zuo J, Niu QW, Frugis G, Chua NH: The WUSCHEL gene promotes vegetative-to-embryonic transition in Arabidopsis. Plant J 2002, 30(3):349-359.

8. Mayer KF, Schoof H, Haecker A, Lenhard M, Jurgens G, Laux T: Role of WUSCHEL in regulating stem cell fate in the Arabidopsis shoot meristem. Cell 1998, 95(6):805-8I5.

9. Sarkar AK, Luijten M, Miyashima S, Lenhard M, Hashimoto T, Nakajima K, Scheres B, Heidstra R, Laux T: Conserved factors regulate signalling in Arabidopsis thaliana shoot and root stem cell organizers. Nature 2007, 446(7/37):8II-8I4. 
10. Wu X, Dabi T, Weigel D: Requirement of homeobox gene STIMPY/WOX9 for Arabidopsis meristem growth and maintenance. Curr Biol 2005, I 5(5):436-440.

II. Riou-Khamlichi C, Menges M, Healy JM, Murray JA: Sugar contro of the plant cell cycle: differential regulation of Arabidopsis D-type cyclin gene expression. Mol Cell Biol 2000 20(I 3):45 I 3-452I.

12. Planchais S, Samland AK, Murray JA: Differential stability of Arabidopsis D-type cyclins: CYCD3; I is a highly unstable protein degraded by a proteasome-dependent mechanism. Plant 2004, 38(4):616-625.

13. Nardmann ], Ji J, Werr W, Scanlon MJ: The maize duplicate genes narrow sheath $I$ and narrow sheath 2 encode a conserved homeobox gene function in a lateral domain of shoot apical meristems. Development 2004, I 3 I ( I 2):2827-2839.

14. Nardmann J, Zimmermann R, Durantini D, Kranz E, Werr W: WOX Gene Phylogeny in Poaceae: A Comparative Approach Addressing Leaf and Embryo Development. Mol Biol Evol 2007 24( I I ):2474-2484.

I5. Richardt S, Lang D, Reski R, Frank W, Rensing SA: PlanTAPDB, a Phylogeny-Based Resource of Plant Transcription-Associated Proteins. Plant Physiol 2007, I43(4): | 452- | 466.

16. Nardmann J, Werr W: The evolution of plant regulatory networks: what Arabidopsis cannot say for itself. Curr Opin Plant Biol 2007, I 0(6):653-659.

17. Philippe H, Laurent J: How good are deep phylogenetic trees? Curr Opin Genet Dev 1998, 8(6):616-623.

18. Theissen G, Kim JT, Saedler H: Classification and phylogeny of the MADS-box multigene family suggest defined roles of MADS-box gene subfamilies in the morphological evolution of eukaryotes. I Mol Evol 1996, 43(5):484-516.

19. Ooka H, Satoh K, Doi K, Nagata T, Otomo Y, Murakami K, Matsubara K, Osato N, Kawai J, Carninci P, et al.: Comprehensive analysis of NAC family genes in Oryza sativa and Arabidopsis thaliana. DNA Res 2003, I 0(6):239-247.

20. Eddy SR: Profile hidden Markov models. Bioinformatics 1998 | 4(9):755-763.

21. Bowman JL, Floyd SK, Sakakibara K: Green genes-comparative genomics of the green branch of life. Cell 2007, I 29(2):229-234.

22. Chandler J, Nardmann J, Werr W: Plant development revolves around axes. Trends Plant Sci 2008, I3(2):78-84

23. Kieffer M, Stern Y, Cook H, Clerici E, Maulbetsch C, Laux T, Davies $B$ : Analysis of the transcription factor WUSCHEL and its functional homologue in Antirrhinum reveals a potential mechanism for their roles in meristem maintenance. Plan Cell 2006, I 8(3):560-573.

24. Hiratsu K, Mitsuda N, Matsui K, Ohme-Takagi M: Identification of the minimal repression domain of SUPERMAN shows that the DLELRL hexapeptide is both necessary and sufficient for repression of transcription in Arabidopsis. Biochem Biophys Res Commun 2004, 32 I(I): I72-178.

25. Tiwari SB, Hagen G, Guilfoyle TJ: Aux/IAA proteins contain a potent transcriptional repression domain. Plant Cell 2004, I 6(2):533-543.

26. Scofield S, Murray JA: KNOX Gene Function in Plant Stem Cell Niches. Plant Mol Biol 2006, 60(6):929-946.

27. Farinas B, Mary C, O Manes C-Ld, Bhaud Y, Peaucellier G, Moreau H: Natural Synchronisation for the Study of Cell Division in the Green Unicellular Alga Ostreococcus tauri. Plant Molecular Biology 2006, V60(2):277-292.

28. Mingam A, Toffano-Nioche $C$, Brunaud $V$, Boudet $N$, Kreis $M$ Lecharny A: DEAD-box RNA helicases in Arabidopsis thaliana: establishing a link between quantitative expression, gene structure and evolution of a family of genes. Plant Biotechnology Journal 2004, 2(5):40I-4I5.

29. Schumaker KS, Dietrich MA: Programmed Changes in Form during Moss Development. Plant Cell 1997, 9(7): I099-I 107.

30. Gagnot S, Tamby J-P, Martin-Magniette M-L, Bitton F, Taconnat L, Balzergue S, Aubourg S, Renou J-P, Lecharny A, Brunaud V: CATdb: a public access to Arabidopsis transcriptome data from the URGV-CATMA platform. Nucl Acids Res 2007:gkm757

31. Meyers BC, Tej SS, Vu TH, Haudenschild CD, Agrawal V, Edberg SB Ghazal H, Decola S: The Use of MPSS for Whole-Genome Transcriptional Analysis in Arabidopsis. Genome Res 2004, |4(8): $164 \mid-1653$
32. Zimmermann P, Hirsch-Hoffmann M, Hennig L, Gruissem W: GENEVESTIGATOR. Arabidopsis Microarray Database and Analysis Toolbox. Plant Physiol 2004, I 36(I):262I-2632.

33. Craigon DJ, James N, Okyere J, Higgins J, Jotham J, May S: NASCArrays: a repository for microarray data generated by NASC's transcriptomics service. Nucl Acids Res 2004 32(suppl_I):D575-577.

34. Caryl AP, Jones GH, Franklin FC: Dissecting plant meiosis using Arabidopsis thaliana mutants. J Exp Bot 2003, 54(380):25-38.

35. Schmid M, Davison TS, Henz SR, Pape UJ, Demar M, Vingron $M$, Scholkopf B, Weigel D, Lohmann JU: A gene expression map of Arabidopsis thaliana development. Nat Genet 2005 , 37(5):50I-506.

36. Li Y, Rosso MG, Viehoever P, Weisshaar B: GABI-Kat SimpleSearch: an Arabidopsis thaliana T-DNA mutant database with detailed information for confirmed insertions. Nucleic Acids Res 2007:D874-878.

37. Siaud N, Dray E, Gy I, Gerard E, Takvorian N, Doutriaux MP: Brca2 is involved in meiosis in Arabidopsis thaliana as suggested by its interaction with Dmcl. Embo / 2004, 23(6): |392-|40I.

38. Richardt S, Lang D, Reski R, Frank W, Rensing SA: PlanTAPDB, a phylogeny-based resource of plant transcription-associated proteins. Plant Physiol 2007, |43(4): |452-| 466.

39. Birnbaum K, Shasha DE, Wang JY, Jung JW, Lambert GM, Galbraith DW, Benfey PN: A Gene Expression Map of the Arabidopsis Root. Science 2003, 302(5652): 1956-1960.

40. Scofield S, Murray JA: The evolving concept of the meristem. Plant Mol Biol 2006, 60(6):V-VII.

4I. Maughan SC, Murray JAH, Bogre L: A greenprint for growth: signalling the pattern of proliferation. Current Opinion in Plant Biology 2006, 9(5):490-495.

42. Tessadori F, Chupeau M-C, Chupeau Y, Knip M, Germann S, van Driel $R$, Fransz $P$, Gaudin V: Large-scale dissociation and sequentia reassembly of pericentric heterochromatin in dedifferentiated Arabidopsis cells. J Cell Sci 2007, I 20(7): | 200- I 208.

43. Raynaud C, Sozzani R, Glab N, Domenichini S, Perennes C, Cella R, Kondorosi E, Bergounioux C: Two cell-cycle regulated SET. domain proteins interact with proliferating cell nuclear antigen (PCNA) in Arabidopsis. Plant J 2006, 47(3):395-407.

44. Hjellstrom M, Olsson ASB, Engstrom P, Soderman EM: Constitutive expression of the water deficit-inducible homeobox gene ATHB7 in transgenic Arabidopsis causes a suppression of stem elongation growth. Plant, Cell \& Environment 2003 26(7): I I27-II36

45. Templeton GW, Moorhead GB: The phosphoinositide-3-OHkinase-related kinases of Arabidopsis thaliana. EMBO Rep 2005, 6(8):723-728.

46. Menand B, Desnos T, Nussaume L, Berger F, Bouchez D, Meyer C, Robaglia C: Expression and disruption of the Arabidopsis TOR (target of rapamycin) gene. PNAS 2002, 99(9):6422-6427.

47. Francis D: The cell cycle in plant development. New Phytologist 1992, I 22(I): I-20.

48. Bernier G: Growth changes in the shoot apex of Sinapis alba during transition to flowering. J Exp Bot I997, 48(5): | $07 \mid-1077$.

49. Jacqmard A, Gadisseur I, Bernier G: Cell Division and Morphological Changes in the Shoot Apex of Arabidopsis thaliana during Floral Transition. Ann Bot 2003, 9 I(5):57 I-576.

50. Svingen T, Tonissen KF: Hox transcription factors and their elusive mammalian gene targets. Heredity 2006, 97(2):88-96.

5I. Jasinski S, Riou-Khamlichi C, Roche O, Perennes C, Bergounioux C, Glab N: The CDK inhibitor NtKISI a is involved in plant development, endoreduplication and restores normal development of cyclin D3; I-overexpressing plants. J Cell Sci 2002, I I 5(5):973-982.

52. Zhou Y, Wang H, Gilmer S, Whitwill S, Keller W, Fowke L: Control of petal and pollen development by the plant cyclin-dependent kinase inhibitor ICKI in transgenic Brassica plants. Planta 2002, 2 I 5(2):248-257.

53. Wang H, Zhou Y, Gilmer S, Whitwill S, Fowke LC: Expression of the plant cyclin-dependent kinase inhibitor ICKI affects cell division, plant growth and morphology. The Plant Journal 2000, 24(5):613-623

54. Altschul SF, Madden TL, Schaffer AA, Zhang J, Zhang Z, Miller W, Lipman DJ: Gapped BLAST and PSI-BLAST: a new generation of protein database search programs. Nucleic Acids Res 1997, 25( I 7):3389-3402. 
55. Samson F, Brunaud V, Duchene S, De Oliveira $Y$, Caboche $M$, Lecharny A, Aubourg S: FLAGdb++: a database for the functional analysis of the Arabidopsis genome. Nucleic Acids Res 2004:D347-350.

56. Derelle E, Ferraz C, Rombauts S, Rouze P, Worden AZ, Robbens S, Partensky F, Degroeve S, Echeynie S, Cooke R, et al:: From the Cover: Genome analysis of the smallest free-living eukaryote Ostreococcus tauri unveils many unique features. PNAS 2006, | 03(3 |): | |647- | |652.

57. Palenik B, Grimwood J, Aerts A, Rouze P, Salamov A, Putnam N, Dupont C, Jorgensen R, Derelle E, Rombauts S, et al:: The tiny eukaryote Ostreococcus provides genomic insights into the paradox of plankton speciation. Proc Natl Acad Sci USA 2007, I 04( I 8):7705-77I0.

58. Taylor JS, Peer $Y$ Van de, Braasch I, Meyer A: Comparative genomics provides evidence for an ancient genome duplication event in fish. Philos Trans $R$ Soc Lond B Biol Sci 200I, 356(I4|4): I66I-1679.

59. Raes J, Rohde A, Christensen JH, Peer $Y$ Van de, Boerjan W: Genome-wide characterization of the lignification toolbox in Arabidopsis. Plant Physiol 2003, I33(3): I05I-I07I.

60. Saitou N, Nei M: The neighbor-joining method: a new method for reconstructing phylogenetic trees. Mol Biol Evol 1987, 4(4):406-425

61. Felsenstein J: Evolutionary trees from DNA sequences: a maximum likelihood approach. I Mol Evol I98I, I 7(6):368-376.

62. Jones DT, Taylor WR, Thornton JM: The rapid generation of mutation data matrices from protein sequences. Comput Appl Biosci 1992, 8(3):275-282.

63. Fitch WM: Toward Defining the Course of Evolution: Minimum Change for a Specific Tree Topology. Systematic Zoology I97I, 20(4):406-4I6.

64. Felsenstein J: PHYLIP: Phylogeny Inference Package (version 3.2). Cladistics 1989, 5:|64-166.

65. Felsenstein J: Confidence limits on phylogenies: An approach using the bootstrap. Evolution 1985, 39:783-79l.

66. Henikoff S, Henikoff JG: Amino acid substitution matrices from protein blocks. Proc Natl Acad Sci USA 1992, 89(22): 10915-10919.

67. Bailey TL, Elkan C: Fitting a mixture model by expectation maximization to discover motifs in biopolymers. Menlo Park, California: AAAI Press; 1994.

68. Crooks GE, Hon G, Chandonia JM, Brenner SE: WebLogo: a sequence logo generator. Genome Res 2004, I 4(6): I I88-I 190.

69. Jaillon O, Aury JM, Noel B, Policriti A, Clepet C, Casagrande A, Choisne N, Aubourg S, Vitulo N, Jubin C, et al.: The grapevine genome sequence suggests ancestral hexaploidization in major angiosperm phyla. Nature 2007, 449(7 I 6 I):463-467.

70. Velasco R, Zharkikh A, Troggio M, Cartwright DA, Cestaro A, Pruss $D$, Pindo M, Fitzgerald LM, Vezzulli S, Reid J, et al.: A high quality draft consensus sequence of the genome of a heterozygous grapevine variety. PLOS ONE 2007, 2(I 2):el 326.

7I. Nishiyama T, Fujita T, Shin IT, Seki M, Nishide H, Uchiyama I, Kamiya A, Carninci P, Hayashizaki Y, Shinozaki K, et al:: Comparative genomics of Physcomitrella patens gametophytic transcriptome and Arabidopsis thaliana: implication for land plant evolution. Proc Natl Acad Sci USA 2003, I00( I3):8007-80I 2.

72. Zhu F, Massana R, Not F, Marie D, Vaulot D: Mapping of picoeucaryotes in marine ecosystems with quantitative PCR of the I 8S rRNA gene. FEMS Microbiology Ecology 2005, 52(I):79-92.

73. Szabados L, Charrier B, Kondorosi A, de Bruijn FJ, Ratet P: New plant promoter and enhancer testing vectors. Molecular Breeding 1995, I(4):419-423.

74. Clough SJ, Bent AF: Floral dip: a simplified method for Agrobacterium-mediated transformation of Arabidopsis thaliana. Plant J 1998, I 6(6):735-743.

75. Bertrand C, Bergounioux C, Domenichini S, Delarue M, Zhou DX: Arabidopsis histone acetyltransferase AtGCN5 regulates the floral meristem activity through the WUSCHEL/AGAMOUS pathway. J Biol Chem 2003, 278(30):28246-2825I.

76. Domenichini S, Raynaud C, Ni D-A, Henry Y, Bergounioux C: AtmndI-[Delta]I is sensitive to gamma-irradiation and defective in meiotic DNA repair. DNA Repair 2006, 5(4):455-464.

77. Nikovics K, Blein T, Peaucelle A, Ishida T, Morin H, Aida M, Laufs P: The Balance between the MIRI64A and CUC2 Genes Con- trols Leaf Margin Serration in Arabidopsis. Plant Cell 2006, I 8( I I):2929-2945.
Publish with Biomed Central and every scientist can read your work free of charge

"BioMed Central will be the most significant development for disseminating the results of biomedical research in our lifetime. "

Sir Paul Nurse, Cancer Research UK

Your research papers will be:

- available free of charge to the entire biomedical community

- peer reviewed and published immediately upon acceptance

- cited in PubMed and archived on PubMed Central

- yours - you keep the copyright 